\title{
Spontaneous and Evoked Glutamate Release Activates Two Populations of NMDA Receptors with Limited Overlap
}

\author{
Deniz Atasoy, ${ }^{1}$ Mert Ertunc, ${ }^{1}$ Krista L. Moulder, ${ }^{3}$ Justin Blackwell, ${ }^{4}$ ChiHye Chung, ${ }^{1}$ Jianzhong Su, ${ }^{4}$ and Ege T. Kavalali ${ }^{1,2}$ \\ Departments of ${ }^{1}$ Neuroscience and ${ }^{2}$ Physiology, The University of Texas Southwestern Medical Center, Dallas, Texas 75390-9111, ${ }^{3}$ Department of \\ Psychiatry, Washington University School of Medicine, St. Louis, Missouri 63110, and ${ }^{4}$ Department of Mathematics, University of Texas at Arlington, \\ Arlington, Texas 76019-0408
}

In a synapse, spontaneous and action-potential-driven neurotransmitter release is assumed to activate the same set of postsynaptic receptors. Here, we tested this assumption using (+)-5-methyl-10,11-dihydro-5H-dibenzo [a,d] cyclohepten-5,10-imine maleate $($ MK801), a well characterized use-dependent blocker of NMDA receptors. NMDA-receptor-mediated spontaneous miniature EPSCs (NMDAmEPSCs) were substantially decreased by MK-801 within 2 min in a use-dependent manner. In contrast, MK-801 application at rest for 10 min did not significantly impair the subsequent NMDA-receptor-mediated evoked EPSCs (NMDA-eEPSCs). Brief stimulation in the presence of MK-801 significantly depressed evoked NMDA-eEPSCs but only mildly affected the spontaneous NMDA-mEPSCs detected on the same cell. Optical imaging of synaptic vesicle fusion showed that spontaneous and evoked release could occur at the same synapse albeit without correlation between their kinetics. In addition, modeling glutamate diffusion and NMDA receptor activation revealed that postsynaptic densities larger than $\sim 0.2 \mu \mathrm{m}^{2}$ can accommodate two populations of NMDA receptors with nonoverlapping responsiveness. Collectively, these results support the premise that spontaneous and evoked neurotransmissions activate distinct sets of NMDA receptors and signal independently to the postsynaptic side.

Key words: synaptic vesicle release; synaptic transmission; synaptic communication; synapse; NMDA receptor; synaptic plasticity; neurotransmission

\section{Introduction}

Spontaneous synaptic vesicle fusion is a salient feature of all synapses (Fatt and Katz, 1952; Del Castillo and Katz, 1954), including those in synaptic networks in vivo (Paré et al., 1997, 1998; Chadderton et al., 2004). These random release events typically activate receptors within a single postsynaptic site and give rise to miniature postsynaptic currents, and therefore, they have been extremely instrumental in analysis of unitary properties of neurotransmission. Under most circumstances, the two forms of release occur concurrently without significant difference in their unitary properties (Isaacson and Walmsley, 1995; Wall and Usowicz, 1998). Spontaneous release typically occurs with a rate of 1-2 vesicles per minute per release site (Geppert et al., 1994; Murthy and Stevens, 1999; Sara et al., 2005), whereas evoked release at individual synapses can occur at an extremely high rate

Received May 30, 2008; revised July 26, 2008; accepted Aug. 20, 2008.

This work was supported by grants from the National Institute of Mental Health to E.T.K. D.A. was supported in part by National Institute of Mental Health Grant MH052804 to Dr. T. C. Südhof. K.L.M. was supported by National Institute on Drug Abuse Grant DA018109. E.T.K. is an Established Investigator of the American Heart Association. We thank K. Huber, H. Krämer, L. Monteggia, T. C. Südhof, J. Sun, D. G. R. Tervo, and members of the Kavalali laboratory for helpful discussions and for critically reading this manuscript. We are grateful to J. Xu and Z. Pang for sharing cultures from synaptotagmin-1-deficient mice. We thank Y. Zhu and C. F. Stevens for the gift of synaptophysinpHluorin construct.

Correspondence should be addressed to Dr. Ege T. Kavalali, Department of Neuroscience, University of Texas Southwestern Medical Center, 5323 Harry Hines Boulevard, Dallas, TX 75390-9111. E-mail: Ege.Kavalali@UTSouthwestern.edu.

DOI:10.1523/JNEUROSCI.2432-08.2008

Copyright $\odot 2008$ Society for Neuroscience $\quad$ 0270-6474/08/2810151-16\$15.00/0
(>100 vesicles per second) (Saviane and Silver, 2006). However, it is still unclear whether spontaneous neurotransmitter release serves a well defined purpose (Otsu and Murphy, 2003; Zucker, 2005). Despite lack of direct evidence for a physiological significance, several studies have shown that the blockade of spontaneous activation of neurotransmitter receptors causes independent or additional downstream effects compared with the blockade of evoked neurotransmission alone (McKinney et al., 1999; Sutton et al., 2004, 2006).

How do postsynaptic neurons distinguish evoked and spontaneous neurotransmission and differentially activate postsynaptic signaling? To address this question, we examined the possibility that spontaneous and evoked neurotransmissions activate nonoverlapping postsynaptic NMDA receptor (NMDAR) populations. For this purpose, our choice to examine NMDAreceptor-mediated synaptic responses was motivated by two reasons. First, recent findings indicate that NMDA receptors signal at rest (Sutton et al., 2006), in addition to their critical role in action-potential-triggered synaptic signaling. Second, working with NMDA responses enabled us to take advantage of $(+)-5$ methyl-10,11-dihydro-5H-dibenzo [a,d] cyclohepten-5,10imine maleate (MK-801), a well characterized use-dependent blocker of NMDA receptors (Huettner and Bean, 1988; Hessler et al., 1993; Rosenmund et al., 1993). In this way, we could show that MK-801-dependent blockade of NMDA-receptor-mediated spontaneous miniature EPSCs (NMDA-mEPSCs) does not cause significant block of subsequent NMDA-receptor-mediated 
evoked EPSCs (NMDA-eEPSCs), and vice versa. Optical imaging of spontaneous and evoked fusion kinetics at individual synapses supported the premise that both forms of release occur from the same synapse. Therefore, we propose that spontaneous and evoked fusion events activate two populations of NMDA receptors with limited overlap, which may enable them to trigger independent postsynaptic signaling events.

\section{Materials and Methods}

Cell culture. High-density-dissociated hippocampal cultures were prepared from 1-d-old Sprague Dawley rat pups or wild-type and syt-1deficient $\left(\right.$ syt- $\left.1^{-/-}\right)$mouse pups using previously described methods (Kavalali et al., 1999). Autaptic hippocampal cultures were prepared as described previously (Mennerick et al., 1995).

Slice preparation. Transverse hippocampal slices $(400 \mu \mathrm{M})$ were prepared from 12- to 21-d-old Sprague Dawley rats and incubated in oxygenated solution containing (in mM) $124 \mathrm{NaCl}, 5 \mathrm{KCl}, 12 \mathrm{NaH}_{2} \mathrm{PO}_{4}, 26$ $\mathrm{NaHCO}_{3}, 10 \mathrm{D}$-glucose, $2 \mathrm{CaCl}_{2}$, and $1 \mathrm{MgCl}_{2}$ at room temperature $\left(24-27^{\circ} \mathrm{C}\right)$. For electrophysiological experiments, after making a cut between CA1 and CA3 regions, individual slices were transferred to the recording chamber that was mounted on the stage of an upright microscope (E600FN; Nikon). During experiments, slices were submerged, and extracellular solution was continuously exchanged with a flow rate of $1-3 \mathrm{ml} / \mathrm{min}$. All experiments were performed at room temperature.

Electrophysiology. In dissociated cultures, cells with pyramidal morphology were whole-cell voltage-clamped to $-70 \mathrm{mV}$ in a modified Tyrode's solution containing the following (in $\mathrm{mm}$ ): $150 \mathrm{NaCl}, 4 \mathrm{KCl}, 2$ $\mathrm{MgCl}_{2}, 10$ glucose, 10 HEPES, and $2 \mathrm{CaCl}_{2}, \mathrm{pH} 7.4$ (310 mOsm). To record and isolate NMDA-receptor-mediated miniature or evoked EPSCs, $\mathrm{MgCl}_{2}$ concentration is reduced to 0 or $0.1 \mathrm{~mm}$ and 6-cyano-7nitroquinoxaline-2,3-dione (CNQX; $10 \mu \mathrm{M}$; Sigma-Aldrich), picrotoxin (PTX; $50 \mu \mathrm{M}$; Sigma), strychnine (1 $\mu \mathrm{M}$; Sigma), and glycine (15 $\mu \mathrm{M}$; Sigma) were added to bath solution. Electrode solution contained the

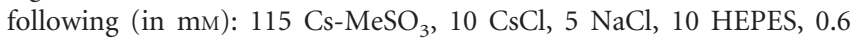
EGTA, 20 tetraethylammonium-Cl, $4 \mathrm{Mg}$-ATP, $0.3 \mathrm{Na}_{2} \mathrm{GTP}$, and $10 \mathrm{li}-$ docaine $\mathrm{N}$-ethyl bromide 2(triethylamino)- $\mathrm{N}$-(2,6-dimethylphenyl) acetamine (QX-314), pH 7.35 (300 mOsm; Sigma). Data were acquired using an Axopatch 200B amplifier and Clampex 8.0 software (Molecular Devices). Recordings were filtered at $2 \mathrm{kHz}$ and sampled at $5 \mathrm{kHz}$. To elicit evoked responses, electrical stimulation was delivered through parallel platinum electrodes in modified Tyrode's solution (duration, $1 \mathrm{~ms}$; amplitude, 20-30 mA). The baseline for the analysis of NMDA-mEPSCs was automatically determined as the average current level of silent episodes during a recording. The events were selected at a minimum threshold of $4 \mathrm{pA}$, and the area under current deflection was calculated to quantify charge transfer. Although in most cases we quantified spontaneous NMDA-mEPSCs as charge transfer, we typically evaluated the reduction in evoked NMDA-eEPSCs in terms of their amplitudes. When we quantified evoked NMDA-eEPSCs as charge transfer (see Figs. 2, 4, 8), this analysis did not result in a significant difference in the estimation of relative block of NMDA-eEPSCs compared with NMDA-mEPSCs, or vice versa.

Whole-cell recordings from autaptic neurons were performed using an Axopatch 1D amplifier, a Digidata 1200 acquisition board, and pClamp software, version 9 (Molecular Devices). Electrodes had resistances of 3-5 M $\Omega$, and access resistance was compensated $80-100 \%$. In all instances, cells were excluded from analysis if a leak current $>300 \mathrm{pA}$ was observed. For recording, the culture medium was exchanged for a saline solution containing the following (in $\mathrm{mm}$ ): $138 \mathrm{NaCl}, 4 \mathrm{KCl}$, $3 \mathrm{CaCl}_{2}, 0 \mathrm{MgCl}_{2}, 10$ glycine, 10 glucose, 10 HEPES, 0.05 bicuculline, and 0.001 NBQX (2,3-dioxo-6-nitro-1,2,3,4-tetrahydro-benzo[f]quinoxaline-7-sulfonamide), $\mathrm{pH}$ 7.25. The whole-cell pipette solution contained (in mM) $140 \mathrm{~K}$-gluconate, $0.5 \mathrm{CaCl}_{2}, 5 \mathrm{EGTA}$, and $10 \mathrm{HEPES}$, $\mathrm{pH}$ 7.25. For synaptic recordings, cells were stimulated with $1.5 \mathrm{~ms}$ pulses to 0 from $-70 \mathrm{mV}$ to evoke transmitter release (Mennerick et al., 1995). Solutions were exchanged via a local multibarrel perfusion pipette with a common perfusion port placed within $0.5 \mathrm{~mm}$ of the cell under study. Liquid junction potential measurements exhibit exchange times between barrels on the order of $\sim 50 \mathrm{~ms}$. NMDA-mEPSCs were recorded in the presence of $500 \mathrm{~nm}$ TTX and analyzed with MiniAnalysis version 6.0 (Synaptosoft).

In hippocampal slices, we recorded from the neurons in the pyramidal cell layer of area CA1 in the whole-cell voltage-clamp configuration. Pipettes were filled with the internal pipette solution that contained (in mM) $110 \mathrm{~K}$-gluconate, $20 \mathrm{KCl}, 10 \mathrm{NaCl}, 10 \mathrm{HEPES}, 0.6 \mathrm{EGTA}, 4 \mathrm{Mg}$ ATP, 0.3 GTP, and 10 QX-314 and that was buffered to pH 7.3 with $\mathrm{CsOH}$ (290 mOsm). eEPSCs were evoked by stimulation (duration, 200 $\mu \mathrm{s}$; amplitude, $10-80 \mu \mathrm{A}$ ) of Schaffer collateral afferents using concentric bipolar tungsten electrodes through a stimulus isolation unit. All statistical comparisons were performed with two-tailed unpaired $t$ test [except in cases in which measurements were paired (see Figs. 2D, 3B)]; values are given as mean \pm SEM.

Fluorescence detection of spontaneous and evoked release kinetics. Highdensity-dissociated cultures were infected with synaptophysin-pHluorin lentivirus at $8 \mathrm{~d}$ in vitro (DIV) and analyzed at 13-14 $\mathrm{d}$ in vitro [synaptophysin-pHluorin construct contained two pHluorins and was a generous gift from Drs. Y. Zhu and C. F. Stevens (Salk Institute, San Diego, CA)]. After recording baseline fluorescence for $1 \mathrm{~min}$, we perfused cultures with $10 \mathrm{~nm}$ freshly prepared folimycin. At high concentrations $(\sim 100 \mathrm{~nm})$, folimycin increased background alkalinization independent of fusion; therefore, in these experiments we used $10 \mathrm{~nm}$ folimycin, which uncovered spontaneous $\mathrm{Ca}^{2+}$-dependent alkalinization as expected from spontaneous fusion. Cultures were then allowed to rest for $10 \mathrm{~min}$ in the presence of $10 \mu \mathrm{M} \mathrm{CNQX}, 50 \mu \mathrm{M} \mathrm{AP}-5$, and $50 \mu \mathrm{M}$ $\mathrm{PTX}$ to estimate spontaneous fusion rate. In these cultures, the presence of CNQX impairs spontaneous action potential firing and thus eliminates the need for TTX application (data not shown). Accordingly, the extent of spontaneous alkalinization in the presence of TTX $\left(2 \mathrm{mM} \mathrm{Ca}^{2+}\right)$ reached $21.2 \%$ of the total pool ( $n=2$ experiments, 70 boutons) compared with $22.4 \%$ of the total pool reached in $2 \mathrm{~mm} \mathrm{Ca}^{2+}$ in CNQX (see Fig. 6). Afterward, cultures were stimulated with parallel field electrodes $(25 \mathrm{~mA}, 1 \mathrm{~ms})$ at a frequency of $1 \mathrm{~Hz}$ for $10 \mathrm{~min}$. At the end of the $10 \mathrm{~min}$ period, cultures were exposed to $8 \mathrm{mM} \mathrm{Ca}^{2+}$ and stimulated maximally at $30 \mathrm{~Hz}$ for 600 pulses to identify functional synaptic vesicle clusters. Images were acquired with a cooled CCD camera (CoolSnapHQ; Roper Scientific) during illumination $(100 \mathrm{~ms})$ at $480 \pm 20 \mathrm{~nm}$ (505 dichroic long pass and $534 \pm 25$ bandpass) via an optical switch (Sutter Instrument) and analyzed using MetaFluor software (Universal Imaging).

Modeling glutamate diffusion in the synaptic cleft and resulting NMDA receptor activation. Our glutamate diffusion model followed the approach previously used by Nielsen et al. (2004) and simulated isotropic diffusion of 4000 glutamate molecules (Xu-Friedman and Regehr, 2003) released from a point source. The glutamate molecules diffuse within a 20 $\mathrm{nm}$ gap of the cleft that has a geometric dimension of $600 \times 600 \times 20 \mathrm{~nm}$. Once they are out of the cleft, they can rapidly diffuse in a large space. The geometry is described by a three-dimensional matrix $S=\left(S_{i, j, k}\right), S_{i, j, k}=1$, if the element is inside the domain of diffusion, and $S_{i, j, k}=0$, if the element is outside of the domain (i.e., in nondiffusible domain).

The standard thermodiffusion equation is applicable for the glutamate concentration $C_{i, j, k}=C\left(x_{i}, y_{j}, z_{k}, t\right)$ as follows:

$$
\frac{\partial C}{\partial t}=D_{\text {glut }}\left(C_{x x}+C_{y y}+C_{z z}\right),
$$

where the diffusion constant $D_{\text {glut }}$ takes the value $0.4 \mu \mathrm{m}^{2} / \mathrm{ms}$ inside the cleft and $0.75 \mu \mathrm{m}^{2} / \mathrm{ms}$ outside the cleft. Using an explicit finite difference scheme, the equation above can be transformed into the following form:

$$
\begin{aligned}
& C^{\prime}{ }_{i, j, k}=C_{i, j, k}+D_{\text {glut }} \frac{d t}{(d x)^{2}}\left(S_{i+1, j, k} C_{i+1, j, k}+S_{i-1, j, k} C_{i-1, j, k}\right. \\
& \left.+S_{i, j+1, k} C_{i, j+1, k}+S_{i, j-1, k} C_{i, j-1, k}+S_{i, j, k+1} C_{i, j, k+1}+S_{i, j, k-1} C_{i, j, k-1}-C_{i, j, k} \sum\right)
\end{aligned}
$$

where

$$
\sum=S_{i+1, j, k}+S_{i-1, j, k}+S_{i, j+1, k}+S_{i, j-1, k} S_{i, j, k+1}+S_{i, j, k-1},
$$


and $C^{\prime}{ }_{i, j, k}=C\left(x_{i}, y_{j}, z_{k}, t+d t\right)$ is the concentration at next time node.

We take $d x=0.01 \mu \mathrm{m}$ and $d t=0.02 \mu \mathrm{s}$. The space step and time step are chosen to ensure that the CFL (Courant-Friedrichs-Lewy) condition (Courant et al., 1928; LeVeque, 2007) is satisfied, and the explicit finite difference scheme will be numerically stable. Once the time course of glutamate distribution is obtained, we use a " $3 \mathrm{C} 2 \mathrm{O}$ " model with twocoupled closed states for receptor kinetics (Popescu et al., 2004) (M-mode NMDA receptor):

$$
\begin{array}{cccccc}
39[C] & 19[C] & 150 & 902 & 4467 & 4630 \\
C^{U} \Leftrightarrow C^{M} \Leftrightarrow C_{1} \Leftrightarrow C_{2} \Leftrightarrow C_{3} \Leftrightarrow & O_{1} \Leftrightarrow O_{2} . \\
58 & 116 & 173 & 2412 & 1283 & 526
\end{array} .
$$

We consider only the M-mode and L-mode kinetics for NMDA receptor. The populations of each of the states are represented by their probability $p_{1}(t), p_{2}(t), p_{3}(t), p_{4}(t), p_{5}(t), p_{6}(t), p_{7}(t)$. The open probability of $p_{\text {open }}(t)=p_{6}(t)+p_{7}(t)$. The populations $p_{\mathrm{i}}(t)$ in M-mode satisfy a system of ordinary differential equations:

$$
\begin{gathered}
\frac{\partial p_{1}}{\partial t}=58 p_{2}-39 C(x, y, z, t) p_{1} \\
\frac{\partial p_{2}}{\partial t}=\left(116 p_{3}-19 C(x, y, z, t) p_{2}\right)-\left(58 p_{2}-39 C(x, y, z, t) p_{1}\right) ; \\
\frac{\partial p_{3}}{\partial t}=-\left(116 p_{3}-19 C(x, y, z, t) p_{2}\right)+\left(173 p_{4}-150 p_{3}\right) ; \\
\frac{\partial p_{4}}{\partial t}=\left(2412 p_{5}-902 p_{4}\right)-\left(173 p_{4}-150 p_{3}\right) ; \\
\frac{\partial p_{6}}{\partial t}=\left(526 p_{7}-4630 p_{6}\right)-\left(1283 p_{6}-4467 p_{5}\right) \\
\frac{\partial p_{7}}{\partial t}=-\left(526 p_{7}-4630 p_{6}\right) .
\end{gathered}
$$

The initial value is $\left.\left(p_{1}(t), p_{2}(t), p_{3}(t), p_{4}(t), p_{5}(t), p_{6}(t), p_{7}(t)\right)\right|_{t=0}=$ $(1,0,0,0,0,0,0)$.

The glutamate concentration $C(x, y, z, t)$ is taken at the location $(x, y$, $z$ ) where the receptor is located (averaged over $10 \mu$ s intervals). For L-mode kinetics, the equations are similar except for the reaction rate constants chosen according to the following kinetic scheme (L-mode NMDA receptor):

$$
\begin{aligned}
& 34[C] \quad 17[C] \quad 127 \quad 580 \quad 2508 \quad 3449 \\
& C^{U} \Leftrightarrow C^{M} \Leftrightarrow C_{1} \Leftrightarrow C_{2} \Leftrightarrow C_{3} \Leftrightarrow O_{1} \Leftrightarrow O_{2} . \\
& \begin{array}{llllll}
60 & 120 & 161 & 2610 & 2167 & 662
\end{array}
\end{aligned}
$$

\section{Results}

\section{NMDA-mEPSCs are rapidly blocked by MK-801}

To test whether spontaneous and evoked synaptic vesicle fusion events activate the same set of postsynaptic receptors, we first pharmacologically isolated NMDA-receptor-dependent synaptic responses by blocking AMPA and GABA receptors in the absence of extracellular $\mathrm{Mg}^{2+}$ and the presence of the NMDA receptor coagonist glycine. We could clearly detect spontaneous NMDAmEPSCs as judged by their sensitivity to AP-5, a selective blocker of NMDA receptors (Fig. 1A). For these experiments, we used dissociated hippocampal cultures because the kinetics of spontaneous synaptic vesicle fusion and recycling are well characterized in this system (Geppert et al., 1994; Ryan et al., 1997; Murthy and Stevens, 1999; Prange and Murphy, 1999; Sara et al., 2005; Virmani et al., 2005).

Application of $10 \mu \mathrm{M}$ MK-801 rapidly blocked spontaneous NMDA-mEPSCs (Fig. 1B). In 12-d-old hippocampal cultures,
MK-801-induced block of NMDA-mEPSCs proceeded with a time constant of $24 \mathrm{~s}$ and reached a plateau within $60 \mathrm{~s}$ (Fig. $1 C, E)$. In these measurements, we quantified charge transfer, a cumulative measure of NMDA receptor activity, because NMDA-mEPSCs are typically noisier than AMPA-mEPSCs (Fig. $1 E$, inset). The kinetics of MK-801 block of spontaneous NMDA$\mathrm{mEPSCs}$ is within a range expected from the previous estimates of the rate of spontaneous vesicle fusion in individual hippocampal synapses in culture (Geppert et al., 1994; Murthy and Stevens, 1999; Sara et al., 2005). The rate of block was further increased in older cultures ( $20 \mathrm{~d}$ in vitro), suggesting that frequency of spontaneous fusion per synapse increases during synapse maturation (Fig. 1D,E). At this developmental stage, the MK-801-induced block reached completion within $30 \mathrm{~s}$. Subsequent application of AP-5, a specific non-use-dependent blocker of NMDA receptors, did not reduce the extent of baseline activity further (quantified as charge transfer) (Fig. $1 F$ ), verifying the completeness of the MK-801 block. In addition, to further test the use-dependent nature of the MK- 801 block of NMDA-mEPSCs, we increased the extracellular $\mathrm{Ca}^{2+}$ from 2 to $8 \mathrm{~mm}$, which causes up to a threefold increase in the rate of spontaneous fusion (Fig. 1G,H) (Sara et al., 2005). In 12-d-old cultures, this increase in extracellular $\mathrm{Ca}^{2+}$ substantially increased NMDA-mEPSC activity as well as AMPAmEPSCs and resulted in a twofold increase in the rate of MK-801 block of NMDA-mEPSCs (Fig. 1I).

In response to glutamate application, NMDA receptors manifest relatively long latencies for opening. In addition, their openings typically outlast the duration of the glutamate pulse. Therefore, MK-801 application is expected to alter the kinetics of NMDA-mEPSCs as they occur (Jahr and Stevens, 1990; Lester et al., 1990; Hessler et al., 1993). In agreement with this expectation, we detected a significant decrease in the decay times of clearly identifiable individual NMDA-mEPSCs after MK-801 application (Fig. $1 J-M$ ). We also observed a small decrease in the rise times of NMDA-mEPSCs as their peaks became sharper in the presence of MK-801. This finding may suggest that individual NMDA receptors open asynchronously in response to glutamate release (Jahr, 1992) (Fig. $1 J, M)$.

To verify that NMDA-mEPSCs were indeed triggered by vesicular glutamate release, we used folimycin, a specific blocker of vacuolar ATPase, which provides the proton gradient required for refilling synaptic vesicles with neurotransmitter. Application of $67 \mathrm{nM}$ folimycin for $15 \mathrm{~min}$ decreased the activity of NMDAmEPSCs as well as AMPA-mEPSCs to a similar extent (supplemental Fig. $1 A, B$, available at www.jneurosci.org as supplemental material), strongly indicating that both types of spontaneous activity were generated by similar vesicle fusion events. This finding makes it unlikely that nonvesicular glutamate release [for instance, of astrocytic origin (Fellin et al., 2004)] could give rise to the NMDA-mEPSCs. This conclusion is also supported by several previous studies that examined the properties of NMDAmEPSCs compared with AMPA-mEPSCs (Umemiya et al., 1999; Groc et al., 2002; Dalby and Mody, 2003). We also evaluated the contribution of silent synapses (that solely contain NMDA receptors) to spontaneous neurotransmission in 20 DIV hippocampal cultures. For this purpose, we compared the frequency of AMPAmEPSCs recorded at $-70 \mathrm{mV}$ to mixed AMPA/NMDA-mEPSCs recorded after relief of $\mathrm{Mg}^{2+}$ block at $+40 \mathrm{mV}$ on a given neuron (supplemental Fig. 1C-E, available at www.jneurosci.org as supplemental material). Under these conditions, we did not detect a significant difference between the frequency of AMPA/NMDAmEPSCs measured at $+40 \mathrm{mV}(4.2 \pm 0.7 \mathrm{~Hz})$ and AMPAmEPSCs measured at $-70 \mathrm{mV}(3.8 \pm 0.5 \mathrm{~Hz})(p>0.05, n=13$ 
High Density Hippocampal Culture
A

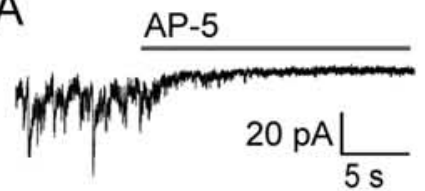

C 12 days in vitro

$0 \mathrm{~s}$. in MK-801

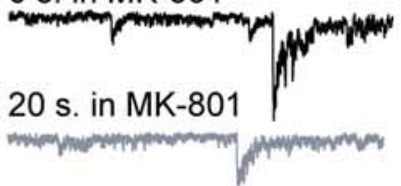

$60 \mathrm{~s}$. in MK-801

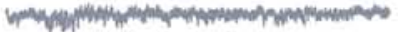

\section{AP5}

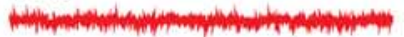

E
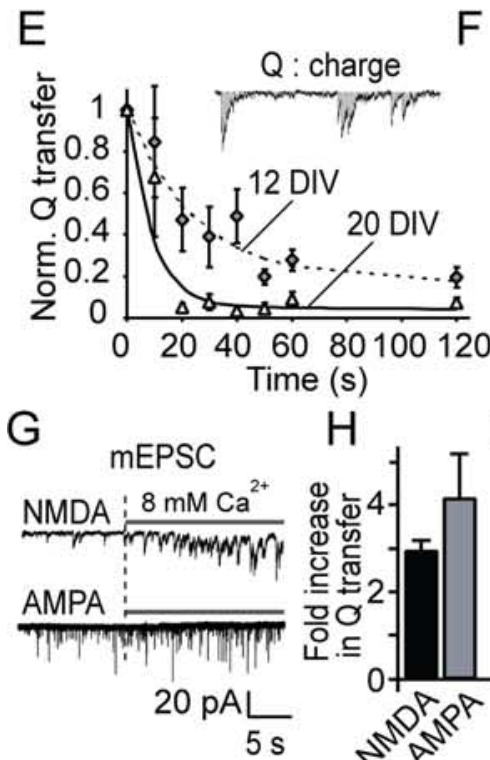

$\mathrm{H}$

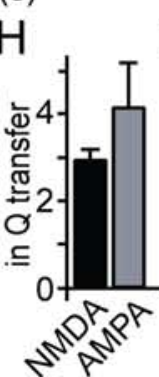

J

NMDA-mEPSC

Before MK-801
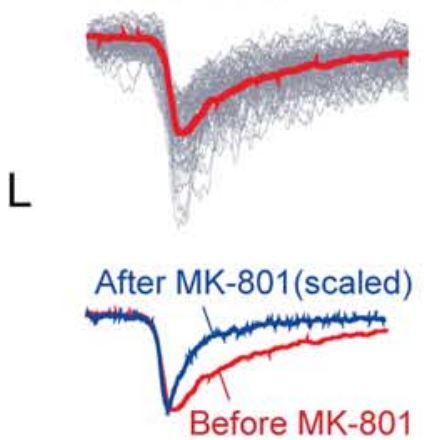

B

D

$\mathrm{F}$
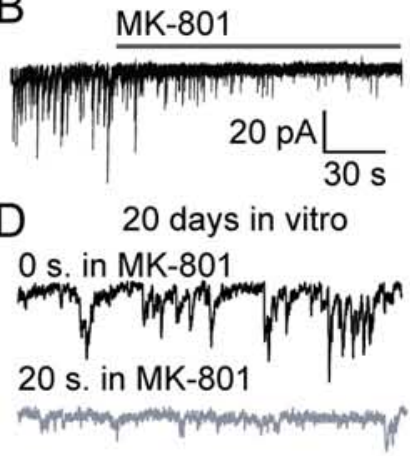

$60 \mathrm{~s}$. in MK-801

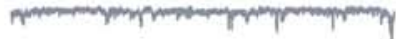

AP5

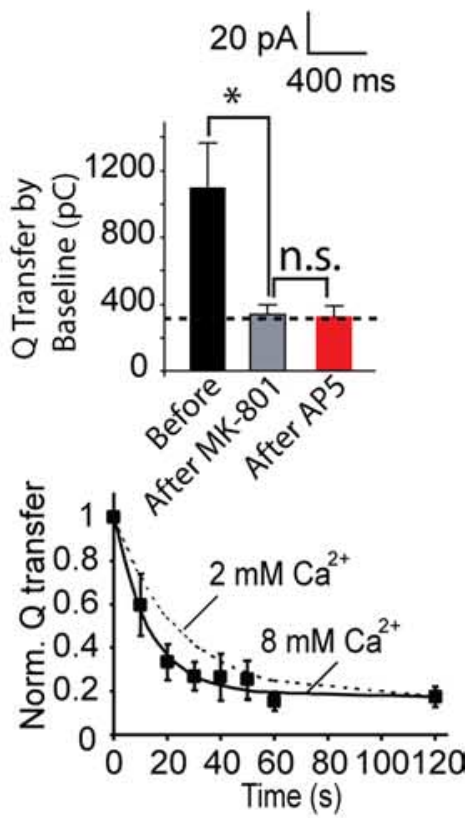

K NMDA-mEPSC in MK-801 $2 \mathrm{~min}$. later

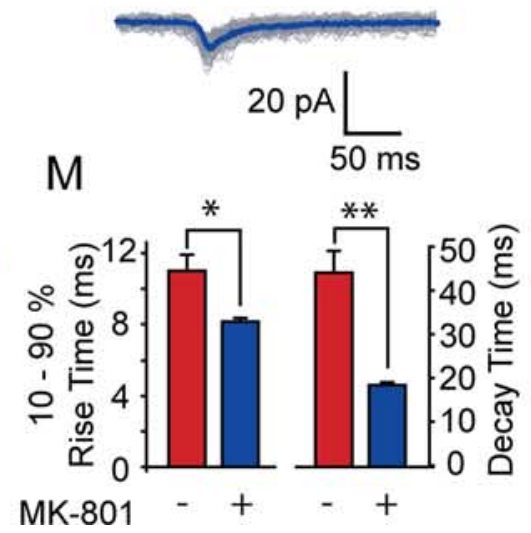

Figure 1. MK-801 block of NMDA-mEPSCs in hippocampal cultures. $\boldsymbol{A}$, NMDA-mEPSCs measured from 20 DIV hippocampal cultures by whole-cell voltage clamp during constant perfusion with extracellular solution containing (in mm) $2 \mathrm{Ca}^{2+}, 0 \mathrm{Mg}^{2+}$, 0.05 picrotoxin, 0.01 CNQX, 0.015 glycine, 0.001 strychnine, and 0.001 TTX. The panel depicts application of $50 \mu \mathrm{m}$ AP-5 during a representative recording. $\boldsymbol{B}$, NMDA-mEPSCs recorded in the same setting as in $\boldsymbol{A}$. The panel depicts application of $10 \mu \mathrm{M}$ MK-801 during a representative recording. $\boldsymbol{C}, \boldsymbol{D}$, Traces show gradual MK-801 block of NMDA-mEPSCs in 12 DIV (C) and 20 DIV (D) cultures ( $n=10$ for each). $\boldsymbol{E}$, The time constant for MK-801 block was faster in 20 DIV $(\tau=9 \mathrm{~s})$ cultures compared with 12 DIV $(\tau=24 \mathrm{~s})$ cells). This finding suggests that NMDAmEPSCs that contribute to the activity at $+40 \mathrm{mV}$ are not significantly in excess of the AMPA-mEPSCs detected in isolation at $-70 \mathrm{mV}$, indicating minimal contribution of silent synapses to spontaneous mEPSC activity in these cultures. In addition, we also tested whether the rapid MK801 block of NMDA-mEPSCs we observed above (Fig. 1) may in part be attributable to block of presynaptic NMDA receptors. To evaluate this possibility, we assessed the impact of rapid extracellular MK-801 application on AMPA-mEPSCs in the absence of $\mathrm{Mg}^{2+}$, and found that the spontaneous fusion rate is only minimally sensitive to presynaptic NMDA receptor activity (for a detailed discussion, see supplemental Fig. 2, available at www. jneurosci.org as supplemental material).

\section{MK-801 treatment at rest minimally affects subsequent evoked NMDA-eEPSCs}

To quantify the cross talk between NMDA receptors that are activated in response to evoked versus spontaneous neurotransmitter release, we triggered release from a large fraction of synapses onto a neuron by invoking a single action-potential-driven NMDA-eEPSC, using field stimulation (Fig. 2A,B). After recording the NMDAeEPSC, we exchanged the medium with a solution containing $10 \mu \mathrm{M}$ MK-801 per 1 $\mu \mathrm{M}$ TTX for 10 min to block spontaneous action potential firing and eliminate NMDA-mEPSCs. At the end of the $10 \mathrm{~min}$

cultures (down to 20\%). In these experiments, NMDA-mEPSC activity was quantified by calculating cumulative charge (Q) transfer in 10 s intervals. $\boldsymbol{F}$, Charge transfer quantified by summation of area below baseline (noise plus events). Before MK$801,1091 \pm 269 \mathrm{pC}$; after $10 \mathrm{~min}$ in MK-801, $337 \pm 55 \mathrm{pC}$; after AP-5, $319 \pm 72 \mathrm{pC}(p=0.016$ before and after MK-801, $p=0.84$ before and after AP-5). $\mathbf{G}$, The effect of elevated extracellular $\mathrm{Ca}^{2+}$ (switch from $2 \mathrm{~mm} \mathrm{Ca}^{2+}$ to $8 \mathrm{~mm} \mathrm{Ca}^{2+}$ ) on NMDA-mEPSCs (top) and AMPA mEPSCs (bottom) at 12 DIV cultures. AMPA mEPSCs are recorded in $2 \mathrm{~mm} \mathrm{Mg}^{2+}, 50 \mu \mathrm{m}$ picrotoxin, $1 \mu \mathrm{M}$ TTX. $\boldsymbol{H}$, Fold increase in mEPSC activity as measured by $Q$ transfer. $I$, The time constant of MK-801 block decreases to $13 \mathrm{~s}$ (from $24 \mathrm{~s}$ ) at $8 \mathrm{~mm} \mathrm{Ca}^{2+}(n=9)$. NMDA$\mathrm{mEPSC}$ traces are superimposed before and after $2 \mathrm{~min}$ of MK801 application. $\boldsymbol{J}, \boldsymbol{K}$, Averaged traces for selected clearly identifiable spontaneous events are superimposed before and 2 min after MK-801 treatment; note the faster decay kinetics of MK-801-exposed miniature events. $L, M$, Comparison of rise and decay times of NMDA-mEPSCs before and 2 min after MK801 treatment. Both rise and decay times are decreased (10 $90 \%$ rise times: before MK-801, $11.2 \pm 0.85 \mathrm{~ms}, n=7$ experiments; after MK-801 treatment, $8.05 \pm 0.2 \mathrm{~ms}, n=7$ experiments; decay times before MK-801, $\tau=43.9 \pm 6.5 \mathrm{~ms}$, $n=7$ experiments; after MK-801 treatment, $\tau=18.8 \pm 0.9$ $\mathrm{ms}, n=7$ experiments). ${ }^{*} p<0.05 ;{ }^{* *} p<0.01$. 


\section{A High Density Hippocampal Culture}

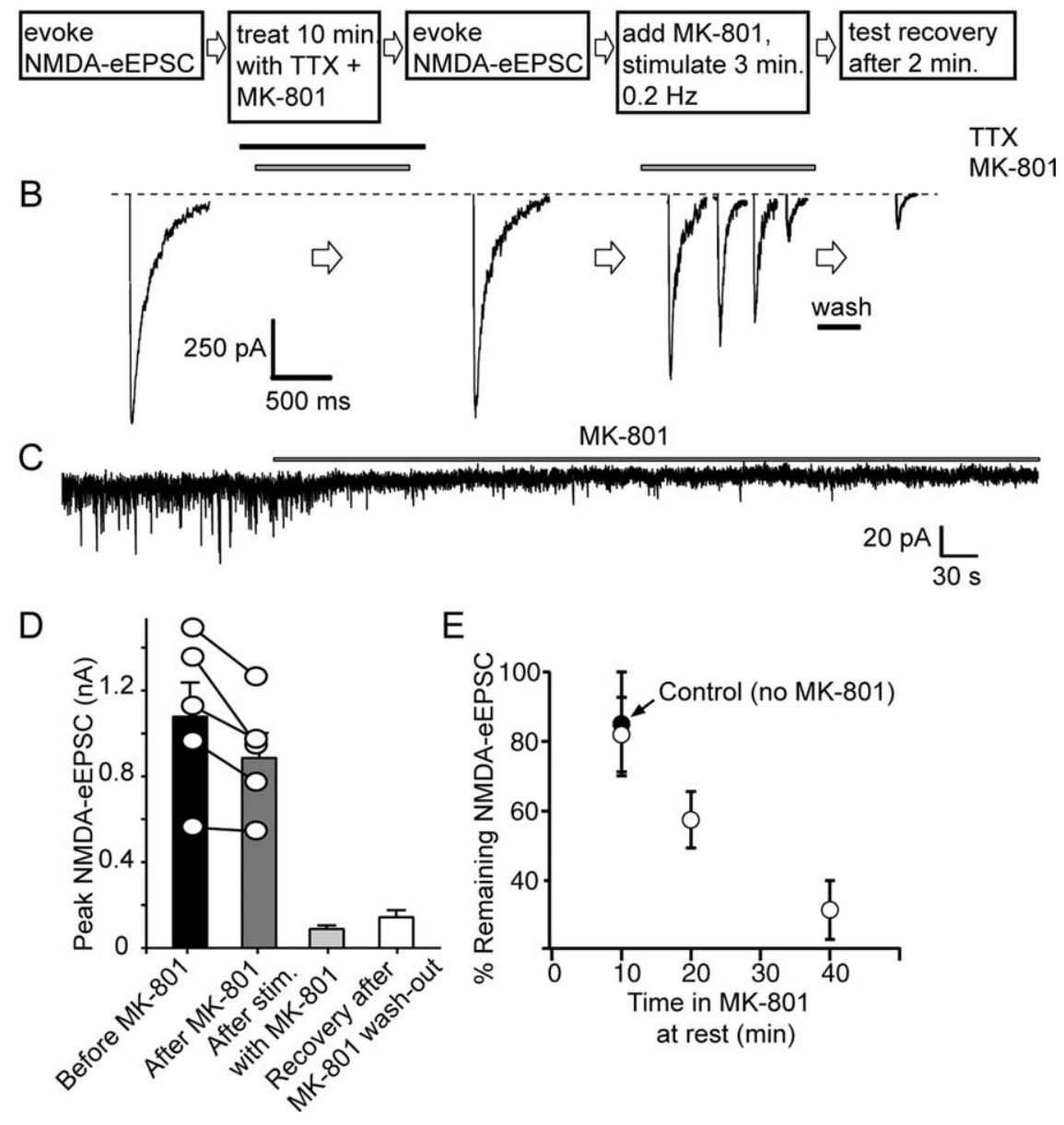

Figure 2. MK-801 treatment at rest minimally affects subsequent evoked NMDA-eEPSCs in hippocampal cultures. $\boldsymbol{A}, \boldsymbol{B}$, Protocol and sample traces of experiments testing cross talk between evoked NMDA-eEPSCs and NMDA-mEPSCs. In 20 DIV cultures, evoked NMDAR-mediated currents are measured with field stimulation by application of $20 \mathrm{~mA}$ pulses at $0.2 \mathrm{~Hz}$ in a solution containing $2 \mathrm{~mm} \mathrm{Ca}^{2+}, 0 \mathrm{~mm} \mathrm{Mg}^{2+}, 50 \mu \mathrm{m}$ picrotoxin, $10 \mu \mathrm{M} \mathrm{CNQX}, 15 \mu \mathrm{m}$ glycine, and $1 \mu \mathrm{m}$ strychnine. This type of stimulation activates all of the synapses formed on a given neuron (as judged by FM dye imaging). The cells are then perfused with TTX for 1 min and with TTX plus MK-801 for $10 \mathrm{~min}$. At the end of a 10 min period, MK-801 is washed out with TTX-containing solution for $1 \mathrm{~min}$, and then TTX is rapidly ( $25 \mathrm{ml} / \mathrm{min}$ ) washed out for $1 \mathrm{~min}$ and NMDA-eEPSCs are again measured $(0.2 \mathrm{~Hz}, 10$ pulses). Afterward, MK-801 is reapplied and cells are stimulated at $0.2 \mathrm{~Hz}$ for 50 pulses until NMDA responses are depressed. At this point, MK-801 is washed out for $2 \mathrm{~min}$, and the test pulse is given to check for any recovery. C, Sample trace for NMDA-mEPSC recorded in between stimulations, in the presence of TTX and MK-801. D, NMDA-eEPSCs do not show significant decrease $(p>0.25)$ after MK-801 treatment (before MK-801, $1072 \pm 157$ pA; after MK-801, $879 \pm 114$ pA). In response to stimulation in the presence of MK-801, they decrease and show no significant recovery after MK-801 washout (after MK-801 plus stimulation, $88 \pm 14$ pA; recovery after MK-801 washout, $141 \pm 33 \mathrm{pA} ; p>0.1)$. $\boldsymbol{E}$, Graph depicting the gradual reduction in NMDA-eEPSC amplitudes after a 10 min MK- 801 treatment $(18 \pm 10 \% ; n=5)$, the decrease under the same conditions without MK- 801 (black circle, $15 \pm$ $5 \% ; n=4$ ), the percentage of reduction in NMDA-eEPSC amplitudes after incubation in MK-801 plus TTX (as well as CNQX and picrotoxin) for $20 \min (42.5 \pm 8.1 \% ; n=5)$ and $40 \min (68.4 \pm 8.4 \% ; n=7)$.

period, we rapidly washed out MK-801 and TTX and measured the size of the evoked NMDA-eEPSC on the same cell. As we have shown above, 10 min perfusion of MK-801 is more than sufficient to block nearly all the NMDA-mEPSC activity on a given neuron (Fig. 2C). In contrast, the amplitudes of NMDA-eEPSCs recorded before and after the incubation period were not affected by the MK-801 treatment in between (before MK-801, $1072 \pm$ $157 \mathrm{pA}$; after MK-801, $879 \pm 114 \mathrm{pA} ; p>0.2, n=5)$, suggesting that application of MK-801 at rest did not block the NMDA receptors activated during stimulation (Fig. $2 D$ ). We also repeated the same experiment using weaker stimulation to decrease peak NMDA-eEPSC by $50 \%$ and activate only a fraction of the synapses. Under this condition, we still observed a modest reduc- tion in NMDA-eEPSC amplitude after 10 min incubation with MK-801 (before MK$801,489 \pm 67 \mathrm{pA} ;$ after MK-801, $411 \pm 29$ $\mathrm{pA} ; p>0.05, n=4)$. Under both strong and weak stimulation conditions, continued stimulation in the presence of MK-801 resulted in rapid use-dependent block of evoked NMDA-eEPSCs. The lack of cross talk between the block of spontaneous NMDA-mEPSCs and evoked NMDAeEPSCs was not caused by unblocking of NMDA receptors during washout of TTX and MK-801 ( $\sim 1 \mathrm{~min})$, because usedependent relief from MK-801 proceeds with a slow time course (Huettner and Bean, 1988). This was also evident in additional experiments, in which a 2 min washout of MK-801 did not induce significant unblocking of the NMDA-eEPSCs (Fig. $2 A, B)$. Here, it is important to note that a 10-min-long whole-cell recording results in some rundown of the NMDA-eEPSC independent of MK-801 treatment. Therefore, the extent of reduction we detected in NMDA-eEPSCs was well within the variability of NMDA-eEPSC amplitudes detected during a stable recording (Fig. 2E, black circle). This finding is consistent with the original experiments, which documented the strict use dependence of MK801 block and proposed the use of this compound to estimate the probability of neurotransmitter release (Hessler et al., 1993; Rosenmund et al., 1993). A small reduction in NMDA-eEPSCs after extended incubation with MK-801 is also in agreement with a recent study, which reported a 28\% decrease in NMDA-eEPSCs after 15 min of MK-801 application (Scimemi et al., 2004).

Previous experiments have also shown that evoked NMDA receptor currents show slow spontaneous recovery $(\sim 20$ min) after MK-801 block (Tovar and Westbrook, 2002), implicating slow mobility of NMDA receptors on dendrites. Indeed, when we incubated hippocampal cultures with MK-801/TTX-containing solution for 20 and $40 \mathrm{~min}$ and compared the sizes of NMDA-eEPSCs to NMDAeEPSCs from vehicle-treated control cultures, we detected a gradual reduction in the size of NMDA-eEPSCs reaching $70 \%$ by 40 min (Fig. 2E). Furthermore, we also detected a faster rate of mixing between the two populations of receptors at $32^{\circ} \mathrm{C}$, presumably because of increased mobility of NMDA receptors. At this temperature, the decrease in NMDA-eEPSCs reached completion within $40 \mathrm{~min}$ after incubation with MK-801 and TTX (supplemental Fig. 3, available at www.jneurosci.org as supplemental material). These results suggest that the NMDA receptors activated by spontaneous release events may mix with other NMDA receptors over long periods, consistent with the slow mobility and mixing of NMDA receptors on a dendrite. In addition, our experiments do not fully exclude other possibilities, such as 
insertion of new NMDA receptors or a very slow rate of spontaneous release at the same location with evoked release.

Cross talk between NMDA-eEPSCs and NMDA-mEPSCs in hippocampal slices We next examined whether these observations obtained in dissociated hippocampal cultures were valid in situ using an acute hippocampal slice preparation. One advantage of the hippocampal slice preparation is that in the absence of stimulation, most of the spontaneous events can be attributed to true spontaneous release even in the absence of TTX. This is because once a cut is introduced between the CA3-CA1 regions of a slice, the propensity of spontaneous action-potential-driven activity diminishes. First, we replicated the original observations in hippocampal slices (Hessler et al., 1993). Namely, a $10 \mathrm{~min}$ application of $50 \mu \mathrm{M}$ MK-801 after an initial evoked NMDA-eEPSC did not occlude the subsequent evoked NMDA-eEPSC in the continued presence of MK-801 (Fig. $3 A, B)$. However, the second evoked NMDA-eEPSC declined rapidly (Fig. $3 A$ ), as expected from the block of open NMDA channels by MK-801 during the NMDAeEPSC. In contrast, spontaneous NMDAmEPSCs detected in the interim period showed swift biphasic block, in agreement with the previous results from the cultures (Fig. 3C,D). The fast component of the decay had a time constant of $19 \mathrm{~s}$, whereas the slow component reached completion by the end of a $10 \mathrm{~min}$ period of MK-801 exposure (down to $4 \%$ of the initial activity). This finding suggests that in slices, the spontaneous fusion rate of $\sim 50 \%$ of the synapses is rather slow $(\tau=245 \mathrm{~s})$ compared with dissociated cultures. After a 10-min-long washout of MK-801, NMDA-mEPSCs showed only $9 \pm 3 \%(n=4)$ recovery, consistent with their successful block during MK-801 application. These findings indicate that in acute hippocampal slices, as in cultures, the NMDA receptor population activated by spontaneous fusion events does not overlap with the one that gives rise to evoked NMDA-eEPSCs.

\section{MK-801 block of NMDA-eEPSCs has minimal effect on} subsequent NMDA-mEPSCs detected on the same cell If the fusion pore kinetics or glutamate release profile of the spontaneous and evoked fusion events in a given synapse differed dramatically, then one can surmise a scenario in which evoked fusion events may reach a higher percentage of receptors, whereas spontaneous fusion events may activate only a very small number of receptors (Cull-Candy and Leszkiewicz, 2004). This setting could give rise to an apparent lack of overlap between the receptor pools activated by the two forms of release, because block of NMDA-mEPSCs would have a negligible effect on evoked NMDA-eEPSCs. To evaluate this possibility, we tested whether use-dependent block of evoked NMDA-eEPSCs impair subsequent NMDA-mEPSCs, using field stimulation in the hippocampal culture system to activate most synapses on a neuron (Fig. $4 A$ ). After recording NMDA-mEPSCs on a given cell, we rapidly perfused MK-801 after washing out TTX and stimulated cultures with 30 action potentials applied at $3 \mathrm{~Hz}(10 \mathrm{~s})$. This moderate stimulation was used to minimize potential glutamate spillover. We then rapidly removed MK-801 and measured the NMDAeEPSC after $1 \mathrm{~min}$ (Fig. $4 \mathrm{~B}$ ). This delay was necessary because this stimulation paradigm induces some synaptic depression independent of the MK-801 block; therefore, the actual extent of block was measured once synapses recover from this weak depression. Under these conditions, we detected a $61.6 \pm 6 \%$ reduction in NMDA-eEPSCs and only a $23.4 \pm 16.5 \%$ reduction in NMDA-mEPSCs (Fig. 4C). Application of MK- 801 for $10 \mathrm{~s}$ without stimulation could by itself induce a $15.5 \pm 6.6 \%$ reduction in NMDA-mEPSCs, indicating that most of the reduction seen in spontaneous NMDA-mEPSCs cannot be attributed to block of evoked release, despite some potential for spillover during the 3 $\mathrm{Hz}$ stimulation (Fig. 4C). In addition, there is some variability in NMDA-mEPSC activity measured over $10 \mathrm{~s}$ intervals $(-2.8 \pm$ $10.9 \% ; n=4)$, which should be taken into account in comparison of these results (Fig. 4C).

\section{Differential use-dependent recovery of NMDA-eEPSCs and} NMDA-mEPSCs from MK-801 block

After removal of MK-801, the recovery of NMDA receptors from MK- 801 block is also use-dependent, because it requires the presence of glutamate and proceeds with a time constant of $90 \mathrm{~min}$ at $-70 \mathrm{mV}$ (Huettner and Bean, 1988). In the next set of experiments, we tested whether NMDA-mEPSCs recover from MK-801 block, and whether this recovery is coupled to recovery of NMDA-eEPSCs. In these experiments, we first measured the ex- 
High Density Hippocampal Culture

A

B
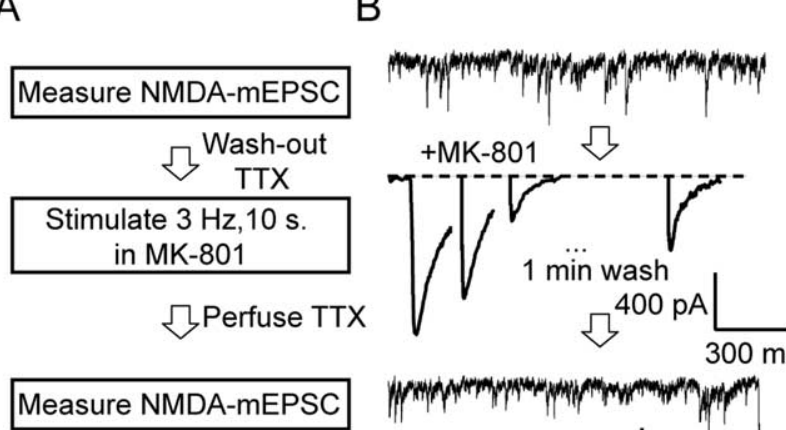

$+\mathrm{MK}-801$

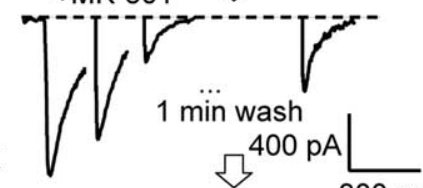

$300 \mathrm{~ms}$
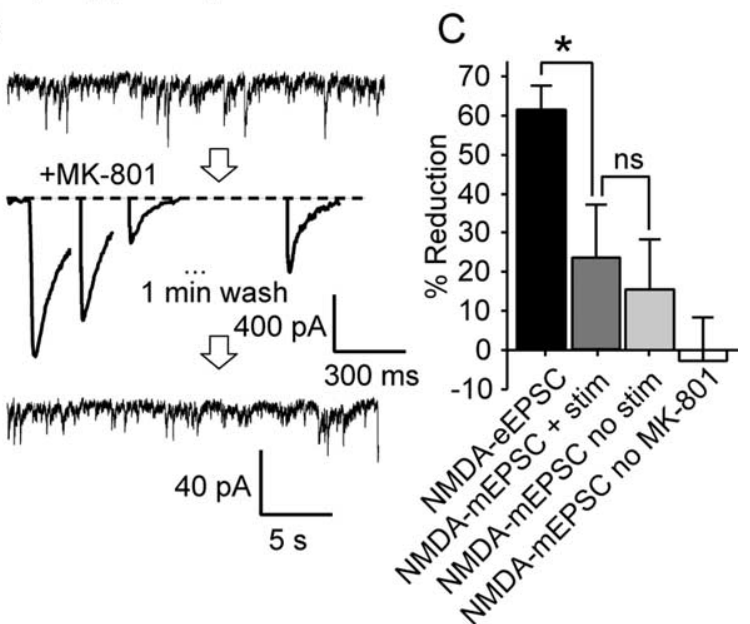

D

$E$

$\mathrm{F}$

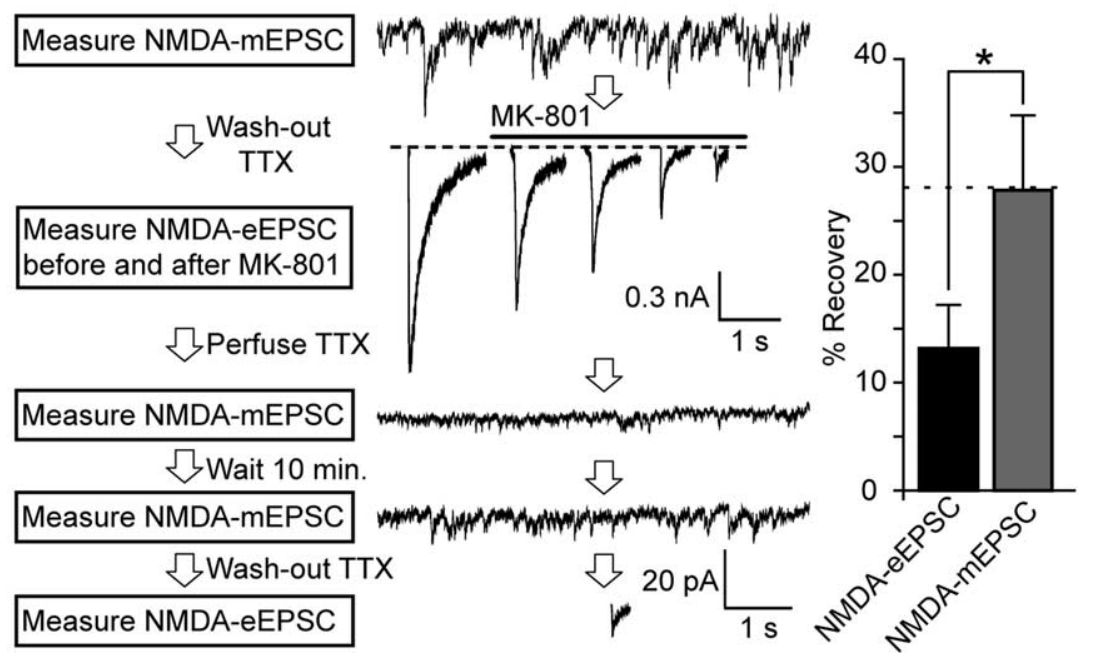

Figure 4. Differential MK-801 block and recovery of NMDA-eEPSCs and NMDA-mEPSCS. $\boldsymbol{A}, \boldsymbol{B}$, Protocol and sample traces from a representative experiment testing cross talk between spontaneous NMDA-mEPSCs and evoked NMDA-eEPSCs. In 20 DIV cultures, NMDA-mEPSCs were measured for $5 \mathrm{~min}$, and then TTX was rapidly washed out for 1 min, followed by MK-801 application. After $3 \mathrm{~Hz}$ stimulation for $10 \mathrm{~s}$, MK-801 was washed out for $1 \mathrm{~min}$, and a test pulse was given to check the level of remaining NMDA-eEPSC amplitude. Finally, TTX was reapplied and NMDA-mEPSCs were measured again. C, Bar graphs showing the percentage of reduction in NMDA-eEPSCs $(61.6 \pm 6 \%)$ and in NMDA-mEPSCs $(23.4 \pm 16.5 \%)\left({ }^{*} p<0.05, n=6\right)$. The percentage of reduction in NMDA-mEPSCs without stimulation $(15.5 \pm 6.6 \% ; n=5)$ was comparable with the decrease seen after stimuIation. NMDA-mEPSCs show some variability in the absence of stimulation or MK-801 $(-2.8 \pm 10.9 \% ; n=4) \cdot \boldsymbol{D}, \boldsymbol{E}$, Experimental protocol and sample traces from the same experiment as in $\boldsymbol{A}$ and $\boldsymbol{B}$, depicting spontaneous recovery from MK-801 block. Evoked NMDA-eEPSCs were measured after TTX washout at $0.2 \mathrm{~Hz}$ stimulation for 10 pulses. MK- 801 was then perfused and neurons were stimulated for another 5 min until evoked responses were diminished. TTX and MK-801 were then perfused to check depression of NMDA-mEPSCs. After $2 \mathrm{~min}$, MK-801 was washed out and NMDA-mEPSCs were recorded for another $10 \mathrm{~min}$. After washout of TTX, evoked NMDA-eEPSCs were measured again to check for spontaneous recovery. $\boldsymbol{F}$, Quantification of percentage of recovery from MK-801 block. NMDA-mEPSC recovery was measured by comparing charge transfer before MK-801 washout to $10 \mathrm{~min}$ after MK-801 removal, whereas evoked recovery was assessed by comparing the last three stimuli during MK-801 application with evoked stimulation after final TTX washout. (NMDA-mEPSC, $27.8 \pm 6.7 \%, n=5$; NMDA-eEPSC, $13.1 \pm 3.9 \%, n=5, p<0.03$ ).

tent of NMDA-mEPSC activity on a given neuron in the presence of TTX and after TTX washout measured evoked NMDA-eEPSCs at $0.2 \mathrm{~Hz}$ stimulation for 10 pulses (Fig. $4 D, E$ ). At this point, we perfused MK-801 during stimulation for another 5 min until evoked responses were diminished. To ensure full block of NMDA-mEPSCs, we reperfused TTX and MK-801 and quantified the depression of NMDA-mEPSCs for $2 \mathrm{~min}$. This procedure guaranteed substantial block of both evoked NMDA-eEPSCs and spontaneous NMDA-mEPSCs. After removal of MK-801, we monitored the recovery of spontaneous NMDA-mEPSCs for 10 min (Fig. 4E). After $10 \mathrm{~min}$, NMDAmEPSCs showed $27.8 \pm 6.7 \%$ recovery, whereas at this time point, evoked NMDA-eEPSCs (measured after TTX washout) had recovered only $13.1 \pm 3.9 \%$ of their initial amplitudes $(n=5, p<0.05)$ (Fig. $4 F$ ). This finding indicates that activation of NMDA receptors at rest by spontaneous glutamate release is not sufficient to remove MK-801 block of NMDA receptors activated in response to evoked release.

To further examine the extent of cross talk between NMDA receptors activated by evoked and spontaneous release, we recorded NMDA-receptor-mediated events in solitary-neuron cultures, in which neurons form recurrent autaptic connections onto themselves. The main advantage of this preparation is that a single action potential generated via intracellular stimulation applied to the cell body of a neuron can access all synapses, thus minimizing the possibility of artifacts that may originate from selective activation of synapses by field stimulation (Mennerick et al., 1995). First, we assessed the effect of blocking NMDA-mEPSCs with MK-801 on subsequent evoked NMDA-eEPSCs (Fig. 5A). eEPSCs and mEPSCs were recorded before a 20 min incubation in $5 \mu \mathrm{M}$ MK-801 and 500 nm TTX (Fig. 5B). At the end of the $20 \mathrm{~min}$ period, MK-801 was washed out and NMDA-eEPSCs and NMDA-mEPSCs were again recorded (Fig. 5A,B). Twenty minutes in MK-801 was sufficient to eliminate almost all spontaneous NMDA-mEPSCs (Fig. 5C); however, similar to high-density hippocampal cultures, the decrease in evoked NMDAeEPSC peak amplitudes was not statistically significant $(p>0.37)$ (Fig. $5 D)$. The percentage of reduction in NMDAmEPSCs was significantly higher than the decrease in NMDA-eEPSCs $(p<0.01)$ (Fig. 5E). Second, we performed the counterexperiment: we first blocked evoked NMDA-eEPSCs with MK-801 and determined whether NMDA-mEPSCs were also affected (Fig. $5 F$ ). For this purpose, we initially recorded mEPSCs for $1 \mathrm{~min}$, just before a 30 pulse stimulus train at $3 \mathrm{~Hz}$ in the presence of MK-801 (Fig. 5G). After the train, MK-801 was washed out for $1 \mathrm{~min}$, and then another evoked NMDA-eEPSC was recorded, followed by the recording of NMDA-mEPSCs for another minute (Fig. $5 G)$. In this setting, we detected a $50.6 \pm 7.2 \%$ decrease in NMDA-eEPSCs (Fig. 5H), whereas the decrease in NMDAmEPSCs was only $24.2 \pm 4.4 \%$ (Fig. $5 I$ ). Although MK-801 application decreased the amplitudes of NMDA-eEPSCs as well as the NMDA-mEPSCs, the percentage of reduction in NMDAeEPSCs was higher than the decrease in NMDA-mEPSCs $(p<$ 0.05 ) (Fig. 5J). This finding is consistent with the experiments 
performed using field stimulation of highdensity cultures (Fig. 4A-C).

Optical analysis of spontaneous and evoked neurotransmission at individual synapses reveals no correlation between the kinetics of the two forms of release If synapses capable of efficient evoked neurotransmitter release were poor in spontaneous release, and vice versa, then one would expect to see a lack of cross talk between the sets of NMDA receptors activated by the two forms of release. In the next set of experiments, we addressed this question by using an optical imaging approach to compare the propensity of evoked and spontaneous release at individual synapses. For this purpose, we infected hippocampal cultures with lentivirus-containing DNA for the synaptic vesicle protein synaptophysin tagged with superecliptic pHluorin (synaptophysin-pHluorin), a GFP (green fluorescent protein)-based $\mathrm{pH}$ sensor that is normally quenched at $\mathrm{pH} 5.5$ within the vesicle lumen, but fluoresces once vesicles fuse and the fluorophore is exposed to extracellular pH 7.4 (Miesenböck et al., 1998). This construct is better localized to synaptic vesicles and manifests minimal surface fluorescence compared with the classical synaptophluorin, which is a fusion construct of synaptobrevin ( $\mathrm{Zhu}$ et al., 2004; Granseth et al., 2006) (Fig. 6A). For these imaging experiments, we selected isolated $1 \mu \mathrm{m}^{2}$ fluorescent puncta, which typically correspond to individual synapses (Liu et al., 1999). To correlate the rate of spontaneous release with evoked release, we incubated cultures with a low concentration of folimycin (10 nM), a high-affinity blocker of vacuolar ATPase required for reacidification of vesicles during endocytosis (Dröse and Altendorf, 1997). Folimycin traps vesicles in an alkaline state after endocytosis and provides a cumulative measure of exocytosis (Ryan et al., 1997; Sankaranarayanan and Ryan, 2001). At rest, using $10 \mathrm{~nm}$ folimycin, we detected a slow $\mathrm{Ca}^{2+}$-dependent increase in fluorescence attributable to spontaneous fusion activity (Fig. $6 A, B)$. At $0 \mathrm{mM} \mathrm{Ca}^{2+}$, the increase in fluorescence was nominal; in contrast, increasing $\mathrm{Ca}^{2+}$ concentration to $8 \mathrm{~mm}$ resulted in a clearly perceptible rise in fluorescence (Fig. $6 \mathrm{~B}$ ). Thus, this spontaneous fluorescence increase was sensitive to alterations in extracellular $\mathrm{Ca}^{2+}$ concentration, similar to spontaneous neurotransmitter release and FM dye release detected in the same system (Fig. 1G,H) (Sara et al., 2005). We also tested this premise by quantifying the rate of fluorescence increase $3 \mathrm{~min}$ after application of saturating stimulation $(30 \mathrm{~Hz}, 2 \mathrm{~min})$ to mobilize most of the recycling vesicles in the presence of folimycin in $2 \mathrm{mM}$ $\mathrm{Ca}^{2+}$. The rate of fluorescence increase attributable to spontane$\left({ }^{*} p<0.05\right)$.

\section{Autaptic Hippocampal Culture}

A

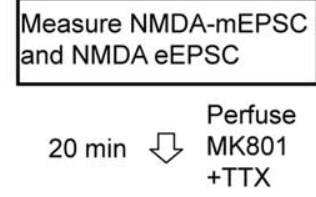

Measure NMDA-mEPSC

Wash-out

MK801

+ TTX

Measure NMDA-mEPSC and NMDA eEPSC

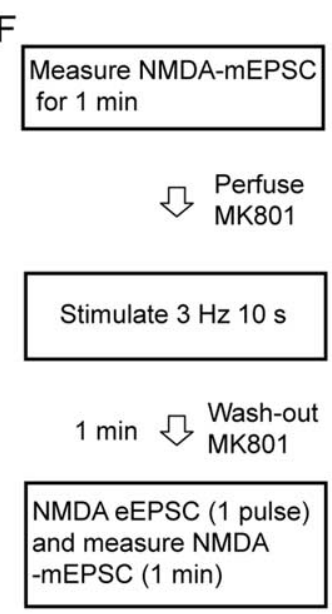

B

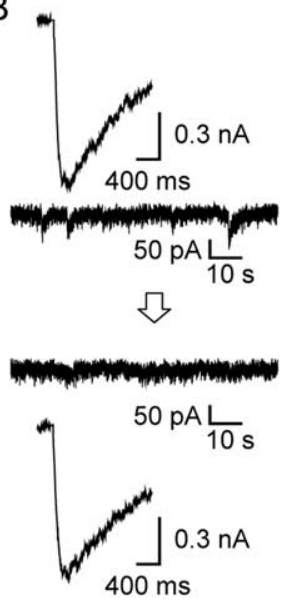

G

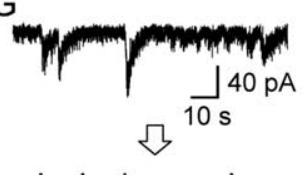

C
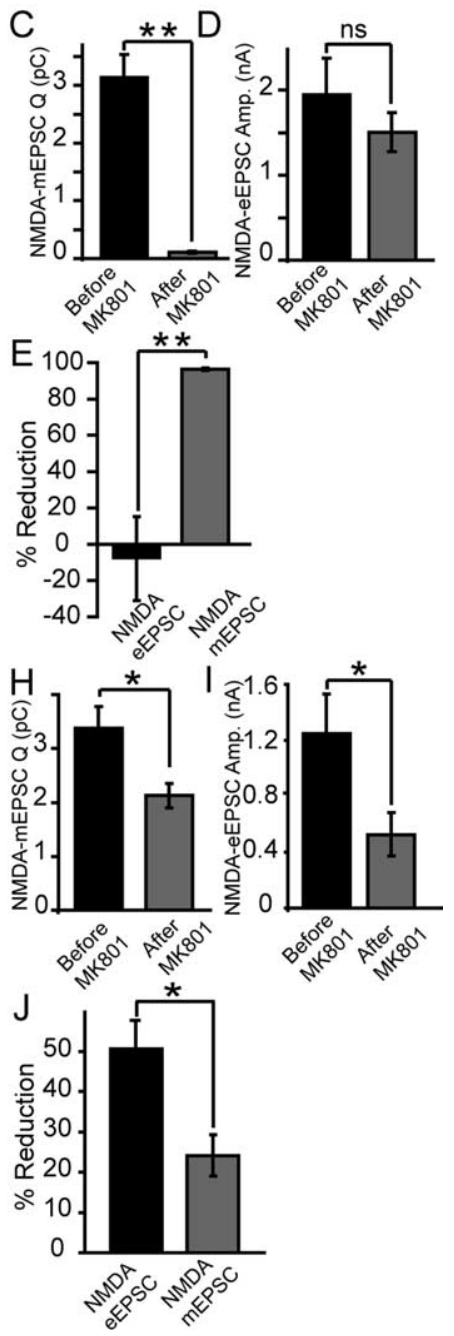

Figure 5. Cross talk between NMDA-eEPSCs and NMDA-mEPSCs in autaptic cultures. $\boldsymbol{A}-\boldsymbol{E}$, Quantification of the effect of blocking NMDA-mEPSCs with MK-801 on subsequent evoked NMDA-eEPSCs $(n=6)$. $\boldsymbol{A}, \boldsymbol{B}$, NMDA-eEPSCs and mEPSCs were recorded before a 20 min incubation in $5 \mu \mathrm{M}$ MK-801 and $500 \mathrm{~nm}$ TTX. At the end of the $20 \mathrm{~min}$ period, MK- 801 was washed out and NMDA-mEPSCs. $\boldsymbol{D}$, In contrast, the decrease in evoked NMDA-eEPSC peak amplitudes was not a statistically significant ( $p>$ 0.37). $\boldsymbol{E}$, The percentage of reduction in NMDA-mEPSCs was significantly higher than the decrease in NMDA-eEPSCs $\left({ }^{* *} p<0.01\right)$ $\boldsymbol{F}-\boldsymbol{J}$, Quantification of the effect of MK-801 block of NMDA-eEPSCs on subsequent NMDA-mEPSCS $(n=8) . \boldsymbol{F}, \boldsymbol{G}, \mathrm{mEPSC}$ were recorded for $1 \mathrm{~min}$, just before a 30 stimuli train at $3 \mathrm{~Hz}$ in the presence of MK-801. After the train, MK-801 was washed out for $\mathrm{min}$, and then another evoked NMDA-eEPSC was recorded, followed by the recording of NMDA-mEPSCS for another minute. $\boldsymbol{H}$, Under these conditions, NMDA-mEPSCs were decreased by $24.2 \pm 4.4 \%\left({ }^{*} p<0.05\right) . I$ I In contrast, the decrease in NMDA-eEPSCS was $50.6 \pm 7.2 \%$. J, The percentage of decrease in NMDA-eEPSCs was significantly higher than the reduction in NMDA-mEPSCs

ous alkalinization under this condition was not significantly different from the alkalinization rate at $0 \mathrm{mM} \mathrm{Ca}^{2+}(p>0.5 ; n=3$ experiments, 366 boutons) (Fig. $6 B$, inset). In addition, the sites of spontaneous fluorescence increase coincided with the locations sensitive to intense action potential stimulation (600 pulses at $30 \mathrm{~Hz}$ ), indicating that these sites corresponded to presynaptic boutons (Fig. 6B).

The kinetics of vesicle mobilization at $1 \mathrm{~Hz}$ is dependent on the probability of neurotransmitter release in individual synapses (Waters and Smith, 2002). This is mainly because at $1 \mathrm{~Hz}$ stimulation there is very little synaptic depression, and thus, each stimulation gives rise to independent trials. Therefore, in the next set of experiments we monitored the rate of spontaneous fluores- 
A High Density Hippocampal Culture
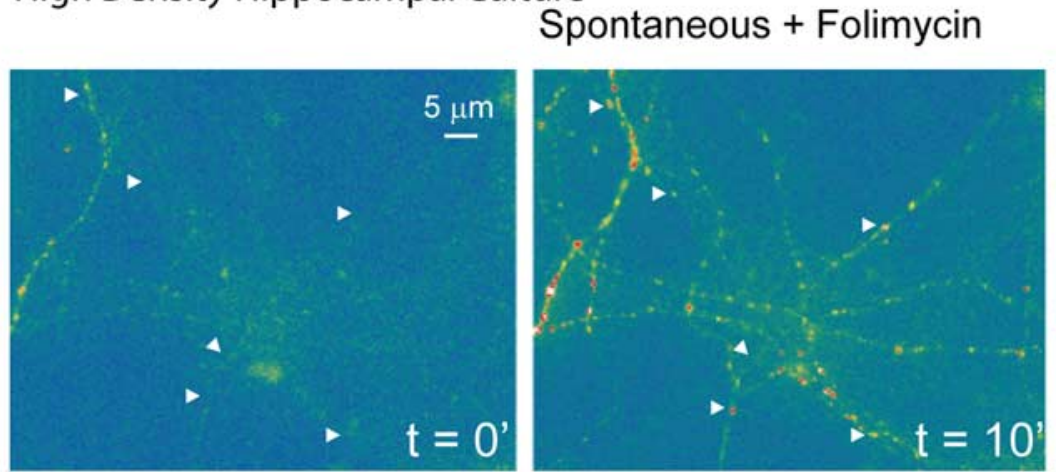

\section{$1 \mathrm{~Hz}+$ Folimycin}

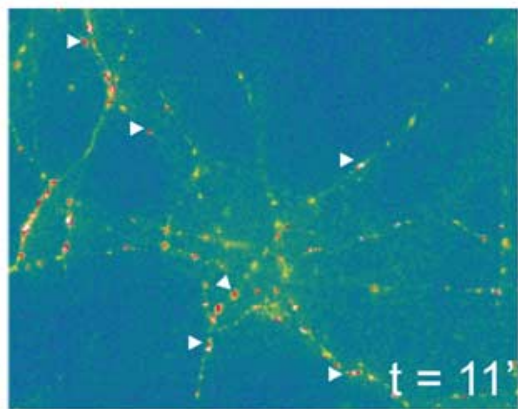

B
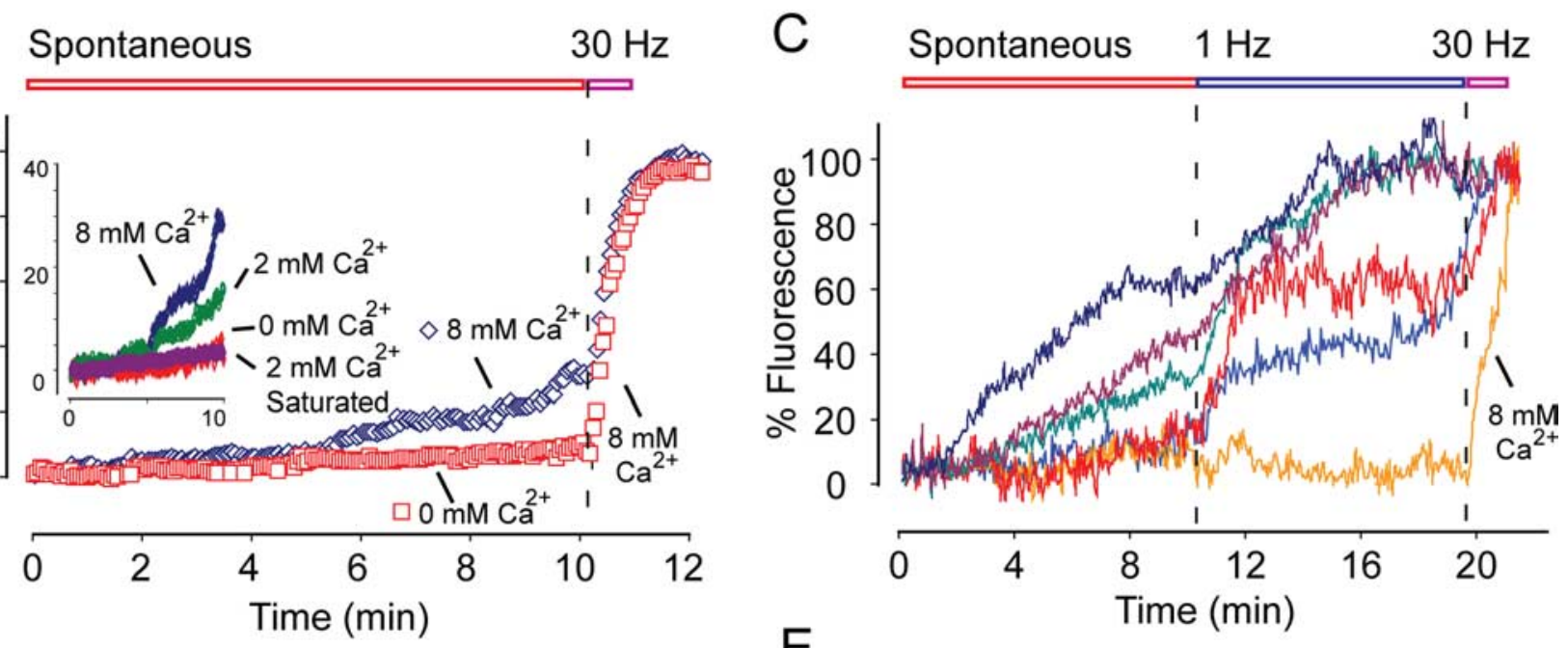

$\mathrm{D}$

\section{Spontaneous $1 \mathrm{~Hz}$}

$30 \mathrm{~Hz}$
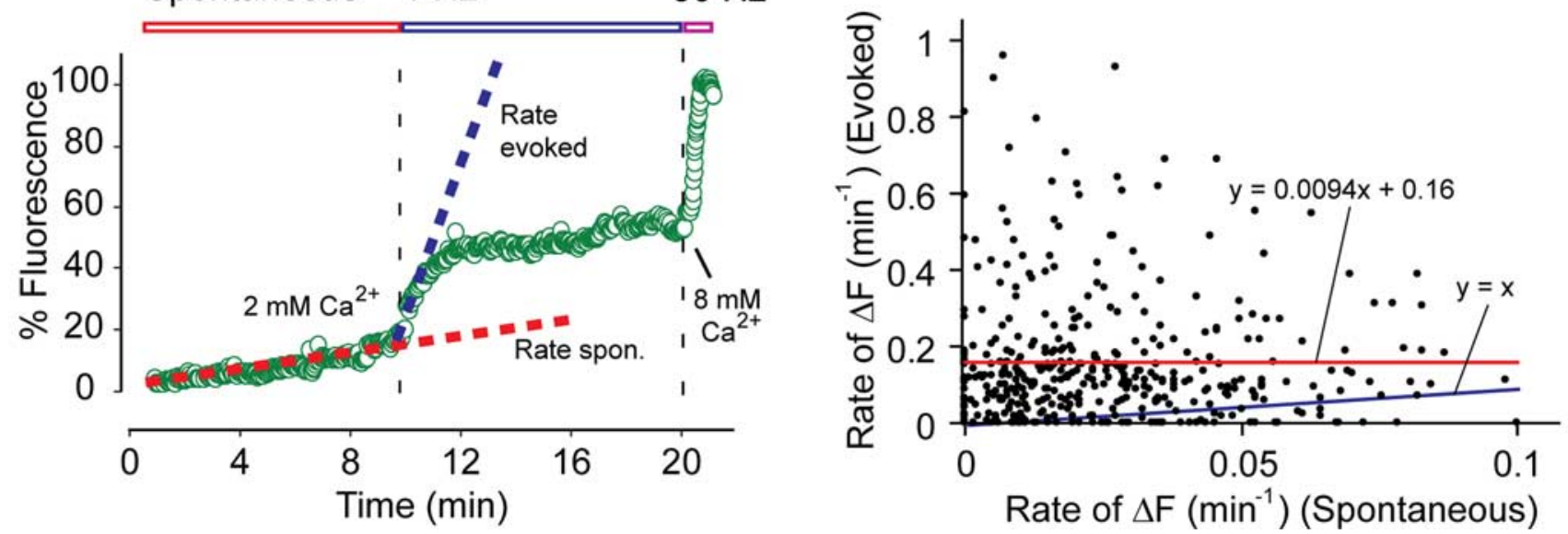

Figure 6. Optical analysis of spontaneous and evoked release with synaptophysin-pHluorin at individual synapses. $A$, Sample images from spontaneous folimycin-dependent alkalinization experiments. Dissociated cultures are infected with synaptophysin-pHluorin lentivirus at $8 \mathrm{DIV}$ and analyzed starting at 13-14 DIV. After baseline imaging for $1 \mathrm{~min}, 10 \mathrm{~nm}$ freshly prepared folimycin

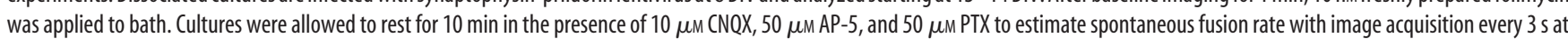
$100 \mathrm{~ms}$ exposure. Arrowheads point to representative boutons selected for analysis. Afterward, cells were stimulated with a $25 \mathrm{~mA}-1 \mathrm{~ms}$ parallel field electrode at $1 \mathrm{~Hz}$ frequency for $10 \mathrm{~min}$. At the end of the 10 min period, cultures were exposed to $8 \mathrm{~mm} \mathrm{Ca}{ }^{2+}$ and stimulated at $30 \mathrm{~Hz}$ for 600 pulses for maximal stimulation to estimate total pool size. Scale bar applies to all three images. $\boldsymbol{B}$, Spontaneous alkalinization rate in $0 \mathrm{~mm} \mathrm{Ca}^{2+}(n=5$ experiments, 166 boutons; reach, $11.3 \%$ of total pool size at the end of $10 \mathrm{~min})$ was substantially slower than in $8 \mathrm{~mm} \mathrm{Ca}^{2+}(n=6$ experiments, 198 boutons; reach, $37.1 \%$ of total pool size at the end of $10 \mathrm{~min})(p<0.01)$. Inset, Comparison of spontaneous alkalinization rates in three different $\mathrm{Ca}^{2+}$ concentrations $(8 \mathrm{~mm}, 2 \mathrm{~mm}, 0 \mathrm{~mm})$ and the same rate $3 \mathrm{~min}$ after saturating stimulation $\left(30 \mathrm{~Hz}, 2 \mathrm{~min}, n=3\right.$ experiments, 366 boutons) in $2 \mathrm{~mm} \mathrm{Ca}{ }^{2+}$. The rate of fluorescence increase after this saturating stimulation was not significantly different from the fluorescence increase at $0 \mathrm{~mm} \mathrm{Ca}^{2+}(p>0.5)$. However, spontaneous alkalinization rates in $8 \mathrm{~mm} \mathrm{Ca}^{2+}$ and in $2 \mathrm{~mm} \mathrm{Ca}^{2+}$ were significantly different from each other as well as from the rate at $0 \mathrm{~mm} \mathrm{Ca}^{2+}(p<0.01)$. We used the slopes of the linear portions of individual traces to calculate the rate of fluorescence rise from spontaneous and evoked recording segments. C, Sample traces from experiments in which spontaneous alkalinization in the presence of $2 \mathrm{~mm} \mathrm{Ca}^{2+}$ was immediately followed by $1 \mathrm{~Hz}$ stimulation to estimate evoked release probability. D, Average of normalized traces in $2 \mathrm{~mm} \mathrm{Ca}^{2+}$ ( $n=9$ experiments, 440 boutons; reach, $22.4 \%$ of total pool size at the end of $10 \mathrm{~min}$ ). $\boldsymbol{E}$, Scatter plot comparing the rate of spontaneous fusion per minute with the rate of fusion evoked at $1 \mathrm{~Hz}$ per minute at the level of individual boutons. The plot could be fitted with a trend line with the equation: $y=0.0094 x+0.16, r=0.001$. $\mathrm{F}$, Fluorescence. 
cence rise in tandem with the rise in fluorescence in response to 1 $\mathrm{Hz}$ stimulation (Fig. 6C,D). This paradigm was followed by $30 \mathrm{~Hz}$ stimulation in the presence of $8 \mathrm{mM} \mathrm{Ca}^{2+}$ to clearly identify synaptic boutons. Under these conditions, we analyzed a total of 440 boutons ( 9 experiments) by measuring the rate of fluorescence rise from the linear portions of individual traces for spontaneous and evoked recording segments (Fig. 6C,D). Restricting our measurements to the linear portions of the traces was necessary because a significant number of the traces reached a plateau, presumably because of reuse of vesicles in an alkaline-trapped state (Fig. 6C).

Overall, the comparison of release rates did not reveal a significant positive or negative correlation between the propensities for evoked and spontaneous fusion of individual synapses $(r=$ 0.001 ) (Fig. 6E). In $14.7 \%$ (65 synapses) of synapses, either no evoked release was exhibited, or evoked release was shown at a rate less than spontaneous release (Kimura et al., 1997). In 79\% of the synaptic boutons, evoked release kinetics was equal to or faster than that of spontaneous release. Six percent ( 27 synapses) did not show any detectable spontaneous release but manifested evoked release. Furthermore, three boutons showed no release under both conditions but responded to $30 \mathrm{~Hz}$ stimulation dramatically. On average, evoked release rate per minute at $1 \mathrm{~Hz}$ was 11.9 times faster than the spontaneous release rate. The median of the fold increase in release rate during $1 \mathrm{~Hz}$ stimulation was 5.4. If we assume an evoked release probability of 0.2 for hippocampal synapses in culture (Murthy et al., 1997), then we would expect release of $\sim 12$ vesicles during $60 \mathrm{~s}$ of $1 \mathrm{~Hz}$ stimulation. From the ratio of evoked and spontaneous release rates, we can estimate the spontaneous release rate for a typical synapse as $1-2$ vesicles per minute. This estimate is in line with earlier work (Geppert et al., 1994; Murthy and Stevens, 1999; Sara et al., 2005), as well as our electrophysiological measurements in this study (Fig. 1E). Together, this analysis suggests that spontaneous and evoked release occur within the same synapses (79\% of the time), however, without significant correlation between their kinetics. In addition, spontaneous release events do not appear to be ectopic, because they occur at discrete spots coincident with intense evoked release rather than being diffusely distributed along an axon.

\section{Differential sensitivity of NMDA-eEPSCs and NMDA- mEPSCs to MK-801 block induced by brief NMDA application}

To further explore the differential properties of NMDA receptors activated in response to evoked versus spontaneous release, we coapplied $100 \mu \mathrm{M}$ NMDA and $30 \mu \mathrm{M}$ MK-801 (in $15 \mu \mathrm{M}$ glycine) to hippocampal cultures and tested the block of global NMDA responses, spontaneous NMDA-mEPSCs, and evoked NMDAeEPSCs (Fig. 7A). Application of NMDA-MK-801 mixture for $5 \mathrm{~s}$ was sufficient to reduce the global NMDA response by $95 \pm 0.6 \%$ $(n=5)$ (Fig. $7 B, C)$. Under the same condition, spontaneous NMDA-mEPSC activity was reduced by $97 \pm 0.8 \%(n=6)$ (Fig. $7 D)$. In contrast, evoked NMDA-eEPSCs were mildly affected by this treatment, because they were only reduced by $45 \pm 8 \%$ ( $n=$ $14 ; 49.6 \pm 5 \%$ in terms of peak amplitudes; mean peak amplitude before MK-801, $1499.8 \pm 197$ pA; after MK-801, $755 \pm 136$ pA) (Fig. $7 E$ ). Subsequent application of AP-5 could completely block this evoked current, whereas it had nominal effect on the remaining baseline activity (data not shown). Furthermore, remaining NMDA-eEPSCs showed minimal recovery from MK-801 block during five pulses applied at $0.1 \mathrm{~Hz}$ (data not shown). Prolongation of the NMDA-MK-801 mixture perfusion to $30 \mathrm{~s}$ caused substantial block of all forms of NMDA receptor activity $(>91 \%$; $n=7)$. Similarly, increasing NMDA concentration to $1 \mathrm{~mm}$ resulted in a substantial block of all NMDA-receptor-mediated activity within $5 \mathrm{~s}(>90 \% ; n=5)$ (Fig. $7 F-I)$. This final result suggests that NMDA concentration was the predominant limiting factor in the partial block of NMDA-eEPSCs consistent with the 10-30 $\mu \mathrm{M}$ affinity of NMDA receptors for NMDA (Patneau and Mayer, 1990). Together, these results indicate that briefly applied exogenous $100 \mu \mathrm{M}$ NMDA has limited ability to activate receptors that mediate evoked NMDA-eEPSCs, whereas NMDA receptors that sustain spontaneous neurotransmission can be readily activated by the same concentration of NMDA and thus strongly blocked by MK-801. These findings are consistent with the premise that NMDA receptors responding to evoked neurotransmission have a low probability for opening compared with the receptors responding to spontaneous neurotransmission. This difference in open probabilities may be attributable to differential subunit composition of receptors responding to evoked and spontaneous release. For instance, NMDA receptors composed of NR1/NR2B subunits display higher affinity for the coagonist glycine and slower deactivation (Priestley et al., 1995; Thomas et al., 2006). However, in a separate set of experiments, we did not detect a significant difference between the compositions of NMDA receptors activated by the two forms of release, because they were both equally blocked by the NR2B-specific antagonist ifenprodil $(3 \mu \mathrm{M})$ by 50\% (NMDA-eEPSCs before ifenprodil, $1412 \pm 299 \mathrm{pA}$; after ifenprodil, $695 \pm 167 \mathrm{pA} ; n=5$, $p=0.019$; NMDA-mEPSCs before ifenprodil, $83.2 \pm 22 \mathrm{pC} / 10 \mathrm{~s}$; after ifenprodil, $47.7 \pm 13 \mathrm{pC} / 10 \mathrm{~s} ; n=5, p=0.003)$. Together, these findings provide additional support to the notion that spontaneous and evoked glutamate release activates distinct populations of NMDA receptors.

\section{Quantitative assessment of geometric constraints on independent NMDA receptor signaling at individual postsynaptic densities}

The results presented so far argue for the coexistence of evoked and spontaneous release within a $1 \mu \mathrm{m}^{2}$ synaptophysinpHluorin-labeled spot that presumably correspond to individual presynaptic terminals. This finding is consistent with earlier work performed using uptake and release of FM dyes (Prange and Murphy, 1999; Sara et al., 2005; Groemer and Klingauf, 2007) and postsynaptic $\mathrm{Ca}^{2+}$ imaging (Murphy et al., 1994; Murthy et al., 2000). Given that the area covered by a single postsynaptic density (PSD) can range between $0.07 \mu \mathrm{m}^{2}(\sim 260 \times 260 \mathrm{~nm})$ to $0.42 \mu \mathrm{m}^{2}(\sim 650 \times 650 \mathrm{~nm})$ (Harris and Sultan, 1995), we next estimated the minimum distance between two sets of NMDA receptors that respond to glutamate release in such a restricted area and have limited cross talk. To address this question, we modeled glutamate diffusion in the synaptic cleft and resulting NMDA receptor activation after fusion of a single vesicle. Our glutamate diffusion model followed the approach previously used by Nielsen et al. (2004). In this model, we simulated isotropic diffusion of 4000 glutamate molecules released from a point source in a simplified synaptic cleft, with a $20 \mathrm{~nm}$ distance between the presynaptic and postsynaptic regions that both cover a $600 \times 600 \mathrm{~nm}$ area $\left(0.36 \mu \mathrm{m}^{2}\right)($ Fig. $8 A, B)$. This large area enabled us to assess glutamate diffusion and NMDA receptor activation at several locations with respect to the initial point of release, and helped us visualize key geometric requirements for limited cross talk between NMDA receptor populations. Within the synaptic cleft, we assumed a diffusion coefficient of 0.4 $\mu \mathrm{m}^{2} / \mathrm{ms}$ in accordance with several earlier estimates (Rusakov and Kullmann, 1998; Xu-Friedman and Regehr, 2003; Nielsen et 


\section{A High Density Hippocampal Culture}

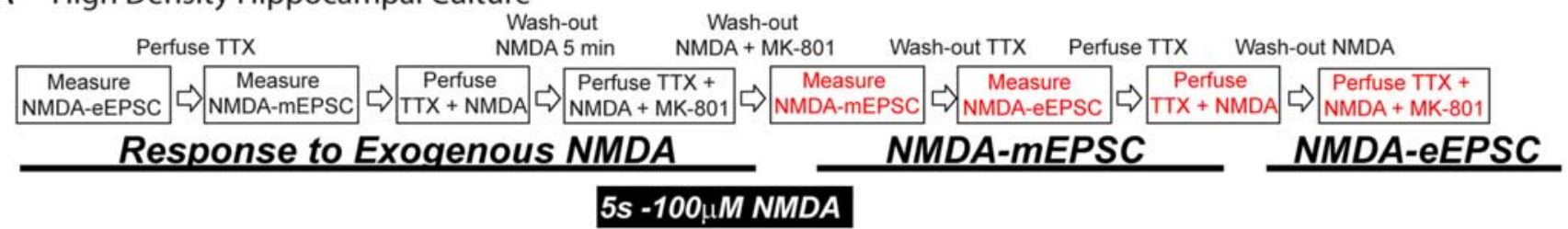
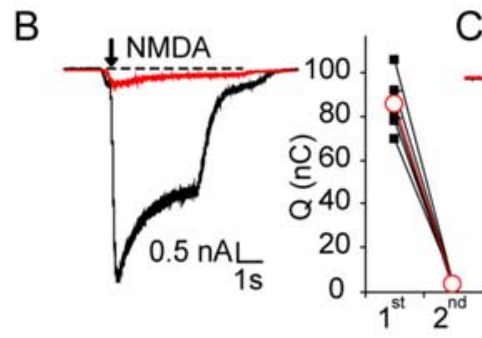

C

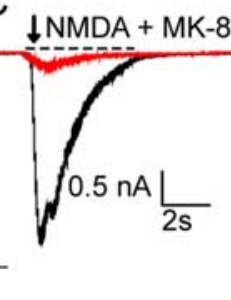

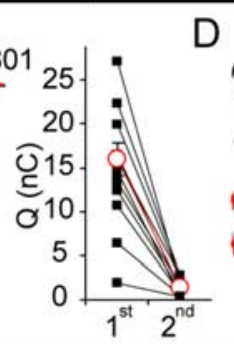

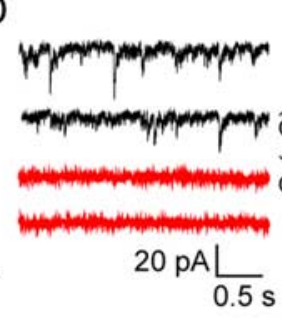

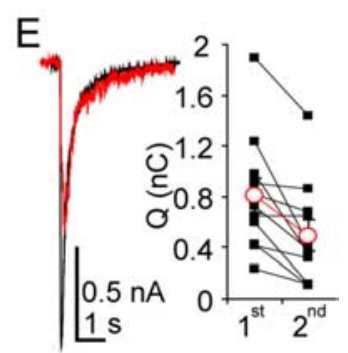

5s - $1 \mathrm{mM}$ NMDA

$\mathrm{F}$

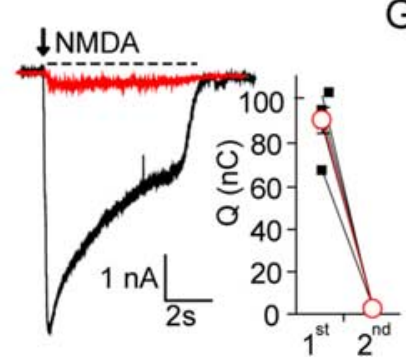

G INMDA+MK-801

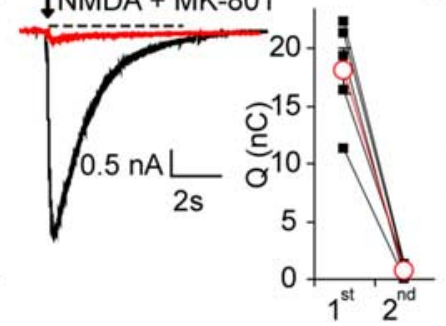

$\mathrm{H}$

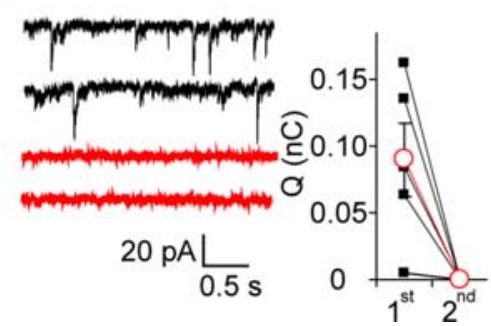

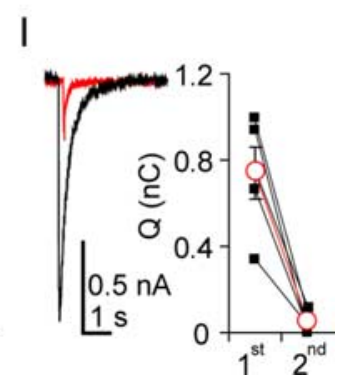

Figure 7. Relatively higher susceptibility of spontaneous NMDA-mEPSCs to MK-801 block during exogenous NMDA application compared with evoked NMDA-eEPSCs. A, Experimental protocol to test susceptibility of evoked NMDA-eEPSCs and spontaneous NMDA-mEPSCs to exogenously applied NMDA and MK-801. Hippocampal neurons from 15 DIV cultures were whole-cell voltage clamped in an external solution similar to the one in Figure 4. First, a baseline for evoked NMDA currents was obtained by $0.1 \mathrm{~Hz}$ stimulation (10 pulses), then TTX was perfused and spontaneous NMDA-mEPSC activity was recorded for $3 \mathrm{~min}$. To obtain an estimate of total NMDA response, $100 \mu \mathrm{m} \mathrm{NMDA}$ containing solution (with $0.5 \mathrm{~mm} \mathrm{Ca}^{2+}$ to minimize possible synaptic release that might be caused by opening of presynaptic NMDA channels) was applied exogenously for $5 \mathrm{~s}$ and rapidly washed out for $5 \mathrm{~min}$ with TTX solution. At the end of the washout, NMDA plus MK-801 (30 $\mu \mathrm{M}$ ) was applied for $5 \mathrm{~s}$. Both NMDA and MK-801 were rapidly washed out for $5 \mathrm{~min}$ with a TTX containing solution, during which the remaining NMDA-mEPSCs were recorded. Then, TTX was washed out and evoked NMDA-eEPSCs were measured to quantify the extent of block. Finally, solutions containing NMDA alone or with MK-801 were applied again to quantify the extent of reduction in the global NMDA response. $\boldsymbol{B}-\boldsymbol{E}$, Sample traces and the quantification of NMDA-receptor-mediated responses during and after $5 \mathrm{~s} 100 \mu \mathrm{M}$ NMDA plus $30 \mu \mathrm{M}$ MK-801 application. $\boldsymbol{B}$, Total NMDA response $\left(5 \mathrm{~s} ; 1^{\text {st }}\right.$, before NMDA plus MK-801; $2^{\text {nd }}$, after NMDA plus MK-801) $\left(1^{\text {st }}, 85.09 \pm 6.25 \mathrm{nC} ; 2^{\text {nd }}, 3.6 \pm 0.44 \mathrm{nC} ; p<0.001, n=5 ;\right.$ percentage of decrease, $\left.95.6 \pm 0.6 \%\right) . C$, NMDA $(100 \mu \mathrm{m})$ plus MK-801 application by integration of charge over $5 \mathrm{s.} 1^{\text {st }}$, Initial application (black trace); $2^{\text {nd }}$, second application (red trace) $\left(1^{\text {st }}, 15.89 \pm 1.89 \mathrm{nC} ; 2^{\text {nd }}, 1.35 \pm 0.33 \mathrm{nC} ; p<0.001, n=14 ;\right.$ percentage of decrease, $90.8 \pm 3 \%)$. D, NMDA-mEPSCs, quantified by charge integration of $20 \mathrm{~s} .\left(1^{\text {st }}, 0.054 \pm 0.025 \mathrm{nc} ; 2^{\text {nd }}, 0.0015 \pm 0.0005 \mathrm{nC} ; p<0.001, n=6 ;\right.$ percentage of decrease, $97.3 \pm$ $0.8 \%)$. $E$, Cumulative charge of evoked NMDA-eEPSCs over $1 \mathrm{~s}\left(1^{\text {st }}, 0.807 \pm 0.13 \mathrm{nC} ; 2^{\text {nd }}, 0.49 \pm 0.11 \mathrm{nC} ; p<0.05, n=14\right.$; percentage of decrease, $\left.45 \pm 8.5 \%\right)$. The decrease in NMDA-mEPSC was significantly higher than the decrease in NMDA-eEPSC, $97.3 \%$ vs $45 \% ; p=0.002$. $\boldsymbol{F}-\boldsymbol{I}$, Similar to $\boldsymbol{B}-\boldsymbol{E}$, but this time NMDA concentration was $1 \mathrm{~mm}$. $\boldsymbol{F}$, Total NMDA response $\left(1^{\text {st }}, 90.95 \pm 6.4 \mathrm{nC}\right.$; $2^{\text {nd }}, 2.35 \pm 0.45 \mathrm{nC} ; p<0.001, n=5$; percentage of decrease, $97.2 \pm 0.6 \%$. G, NMDA (1 mM) plus MK-801 ( $1^{\text {st }}, 18.14 \pm 1.9 n C ; 2^{\text {nd }}, 0.7 \pm 0.27 n c ; p<0.001, n=5 ;$ percentage of decrease, $96.3 \pm 1 \%) . H$, NMDA-mEPSCS $\left(1^{\text {st }}, 0.08 \pm 0.026 \mathrm{nc} ; 2^{\text {nd }}, 0.0002 \pm 0.0001 \mathrm{nc} ; p<0.001, n=5 ;\right.$ percentage of decrease, $\left.99.7 \pm 0.21 \%\right) . I$, Evoked NMDA-eEPSC $\left(1^{\text {st }}, 0.74 \pm 0.11 \mathrm{nC} ; 2^{\text {nd }}\right.$, $0.055 \pm 0.022 \mathrm{nc} ; p<0.001, n=5$; percentage of decrease, $91 \pm 2 \%$ ). The decrease in NMDA-mEPSC was not significantly different from the decrease in NMDA-eEPSC; $p>0.1$. Q, Charge.

al., 2004), whereas outside the cleft we assumed a diffusion coefficient of $0.75 \mu \mathrm{m}^{2} / \mathrm{ms}$. The latter represents the diffusion coefficient for glutamate in free solution (Xu-Friedman and Regehr, 2003). Thus, once glutamate molecules leave the cleft, they can diffuse in a large region with a faster rate. In this model, we did not incorporate glutamate buffering by transporters, because our experiments showed that application of $50 \mu \mathrm{M}$ threo- $\beta$ benzyloxyaspartate (TBOA), a potent wide spectrum blocker of glutamate transporters, did not lead to a cross talk between spontaneous NMDA-mEPSC activity and subsequent evoked NMDA-eEPSCs (10 min of incubation with TTX plus MK-801 plus TBOA decreased NMDA-eEPSCs only to $97.1 \pm 11 \%$ of their initial amplitude; $n=5$ ). However, TBOA treatment caused a $3.18 \pm 07$-fold increase in NMDA-mEPSCs $(n=5)$, suggesting that these transporters are active and may limit glutamate diffusion at rest.

To estimate the degree of NMDA receptor activity at various locations, we equally spaced 16 NMDA receptors within the 0.36 $\mu \mathrm{m}^{2}$ PSD area (Fig. $8 A, \mathrm{R} 1$ through R16). Figure $8 C$ depicts the decrease in peak glutamate concentration after release of a single vesicle on top of receptor 6 (R6; a receptor located near the center of the PSD) versus glutamate release on R16 (a receptor at the periphery of the PSD). The distance between these receptors is $466 \mathrm{~nm}$, and thus, they are located at the opposite edges of a $\sim 0.2$ $\mu \mathrm{m}^{2}$ area. Regardless of the release site (on R6 or R16), glutamate concentration decreases more than a 1000 -fold once it reaches the other receptor (Fig. 8C).

To determine the peak open probabilities of NMDA receptors after glutamate release on R6 or R16, we used the NMDA receptor activation scheme proposed by Popescu et al. (2004). This scheme can account for several key features of synaptic NMDAreceptor-mediated currents, including their nonsaturation behavior seen in response to repetitive stimulation, as well as their predominant modes of activity (high open probability $\mathrm{H}$-mode, medium open probability M-mode, and low open probability L-mode) (Popescu et al., 2004). Figure 8 D depicts the decrease in open probabilities of receptors R16 through R1 after release on R16 (in M-mode or L-mode). This plot indicates that receptors at 
the opposite corner of the PSD at a distance of $466 \mathrm{~nm}$ and beyond (e.g., R13, R9, and R6 through R1) are far less likely to open (at least nine-fold) in response to glutamate release on R16 (with the assumption that all receptors possess the same mode of activity, e.g., all in M-mode or L-mode). However, if these receptors respond to evoked release and typically manifest a low-probability mode (such as the L-mode), as suggested by our experiments presented in Figure 7, and spontaneous release occurs in the vicinity of R16 (near the periphery of the PSD), then the receptors at the opposite corner of the PSD show a more than $\sim 20$-fold reduction in peak open probability compared with R16. This dramatic difference in peak open probabilities of the receptor populations can explain the finding that application of MK-801 at rest has very little effect on subsequent evoked responses (Figs. 2, 3, 5A-E).

We next estimated the open probabilities of all receptors in response to glutamate release onto R6, which according to our model is the putative site of evoked release (Fig. $8 E$ ). In this case, if all receptors are in the same mode, then R16 displays a 4.5-fold decrease in open probability compared with R6. However, the difference between the open probabilities of R16 and R6 decrease to $\sim 1$.9-fold if we follow the above assumption (and our results presented in Fig. 7) that R16 is M-mode and R6 is L-mode. This difference in peak open probabilities is compatible with the results of experiments in which block of evoked NMDA responses results in a milder reduction of subsequent spontaneous NMDA receptor activity (Fig. $4 A-C, 5 F-J$ ).

Together, this model can recapitulate several key features (including the asymmetry in the extent of cross talk detected after MK-801 block of NMDA-mEPSCs vs NMDA-eEPSCs) with the assumption that within a $0.36 \mu \mathrm{m}^{2}$ PSD, a release event near the center (e.g., the vicinity of R6) represents evoked neurotransmission, whereas a fusion event at the periphery of the PSD (e.g., near R16) corresponds to spontaneous release. Moreover, this model indicates that our experimental findings are in line with the commonly accepted parameters governing glutamate diffusion in synapses (Rusakov and Kullmann, 1998; Xu-Friedman and Regehr, 2003; Nielsen et al., 2004). According to this model, medium to large $\left(>0.2 \mu \mathrm{m}^{2}\right.$ area $)$ synapses can easily accommodate independent signaling via spontaneous and evoked release with some geometric constraints. In a mediumsized synapse, the two forms of release would need to be organized laterally (toward opposite corners of the synaptic cleft), whereas in a large synapse the independence of spontaneous and evoked neurotransmission can be achieved if the two forms of release are organized in a center-surround manner. This model does not fully exclude the possibility that some synapses, especially small synapses $\left(<0.2 \mu \mathrm{m}^{2}\right.$ area $)$ that may be below the
B
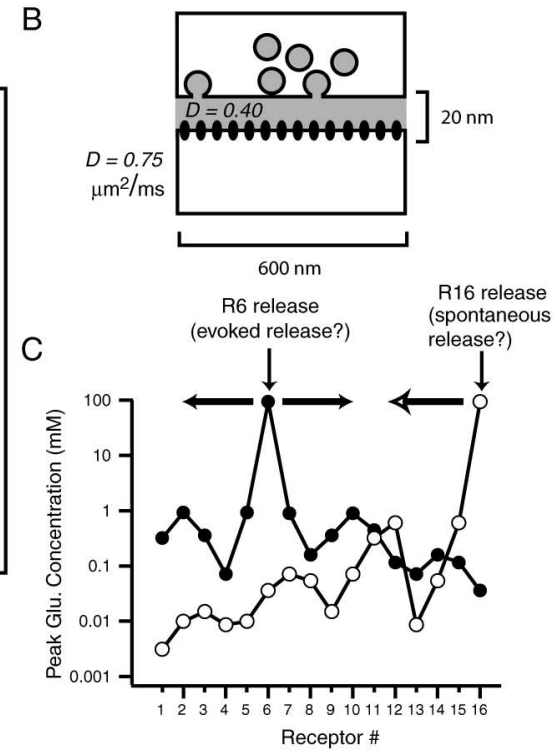

E

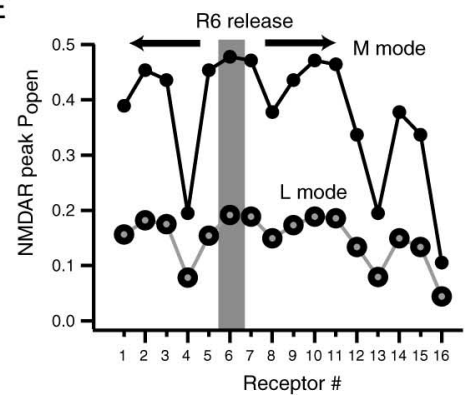

Figure 8. Modeling glutamate diffusion reveals geometric constraints on independent NMDA receptor signaling in individual glutamate molecules released from a point source in a simplified synaptic cleft, with a $20 \mathrm{~nm}$ distance between the activity at various locations, we equally spaced $16 \mathrm{NMDA}$ receptors (R1 through R16) within the $0.36 \mu \mathrm{m}^{2}$ PSD area. Within the assess glutamate diffusion and NMDA receptor activation at several locations with respect to the initial point of release. $C$ Graph depicts the decrease in peak glutamate concentration after release of a single vesicle on top of R6 (a receptor located near the center of the PSD) versus glutamate release on $\mathrm{R} 16$ (a receptor at the periphery of the PSD). The distance between these receptors is $466 \mathrm{~nm}$. $\boldsymbol{D}, \boldsymbol{E}$, Graphs show the peak open probabilities of NMDA receptors after glutamate release on R6 or R16. Open lities are estimated using the NMDA receptor activation scheme proposed by Popescu et al. (2004). This scheme incorpoprobability L-mode). $\boldsymbol{D}$, The decrease in open probabilities of receptors R16 through R1 (in M-mode or L-mode) after release on $R 16$, the putative site of spontaneous release. $E$, The open probabilities of all receptors in response to glutamate release onto R6, which according to our model is the putative site of evoked release.

resolution of our optical experiments (shown in Fig. 6), can manifest solely spontaneous or evoked release and contribute to our electrophysiological observations. Furthermore, we should note here that deviations from the common assumptions we used in this model (such as a decrease in the number of glutamate molecules per vesicle, the peak glutamate concentration in the cleft after fusion, incorporation of glutamate transporters, or other glutamate binding sites) could have a profound effect on the extent of cross talk between the two forms of release.

Asynchronous release detected in the absence of synaptotagmin 1 or in the presence of $\mathrm{Sr}^{2+}$ is resistant to MK801 application at rest

Finally, we extended our observations by asking whether asynchronously released neurotransmitter quanta activate the same set of 


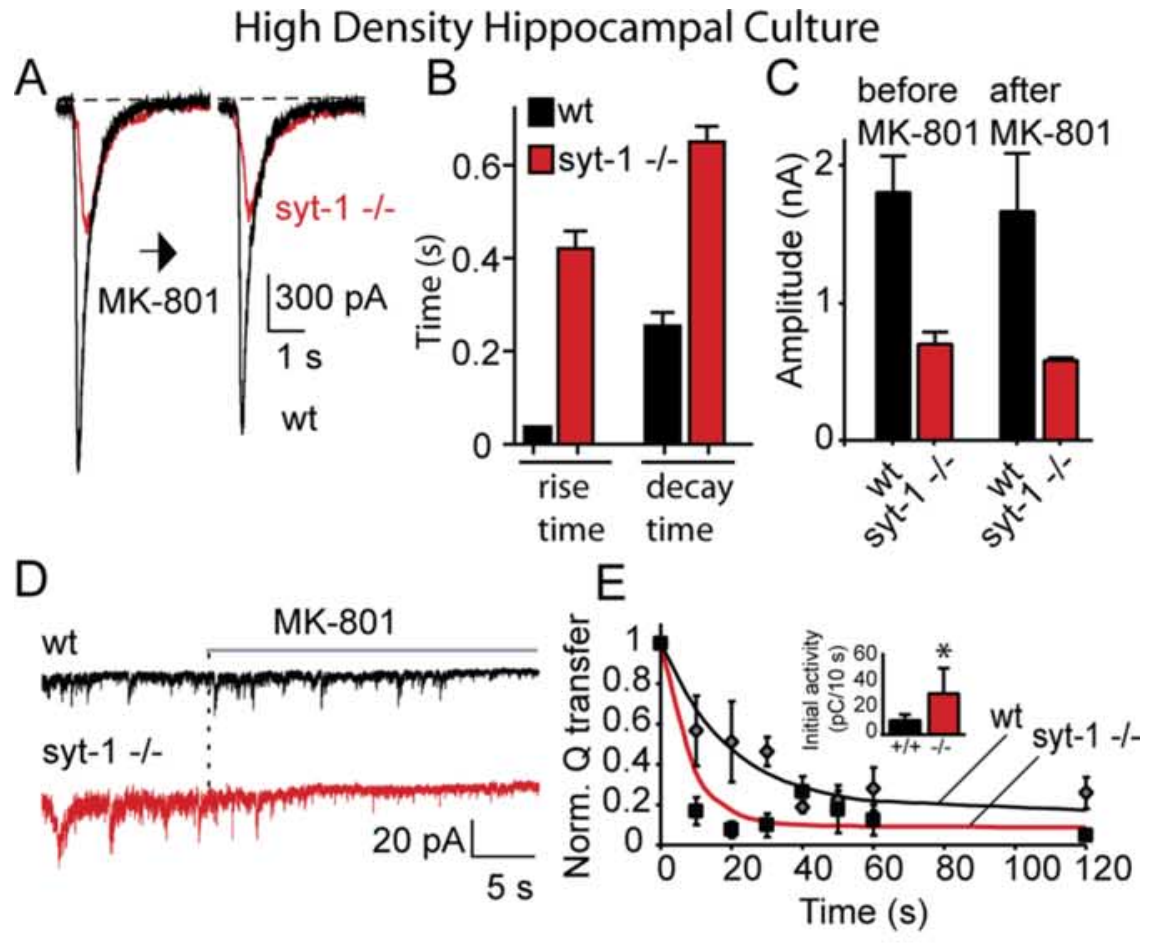

$\mathrm{F}$

AMPA-eEPSC
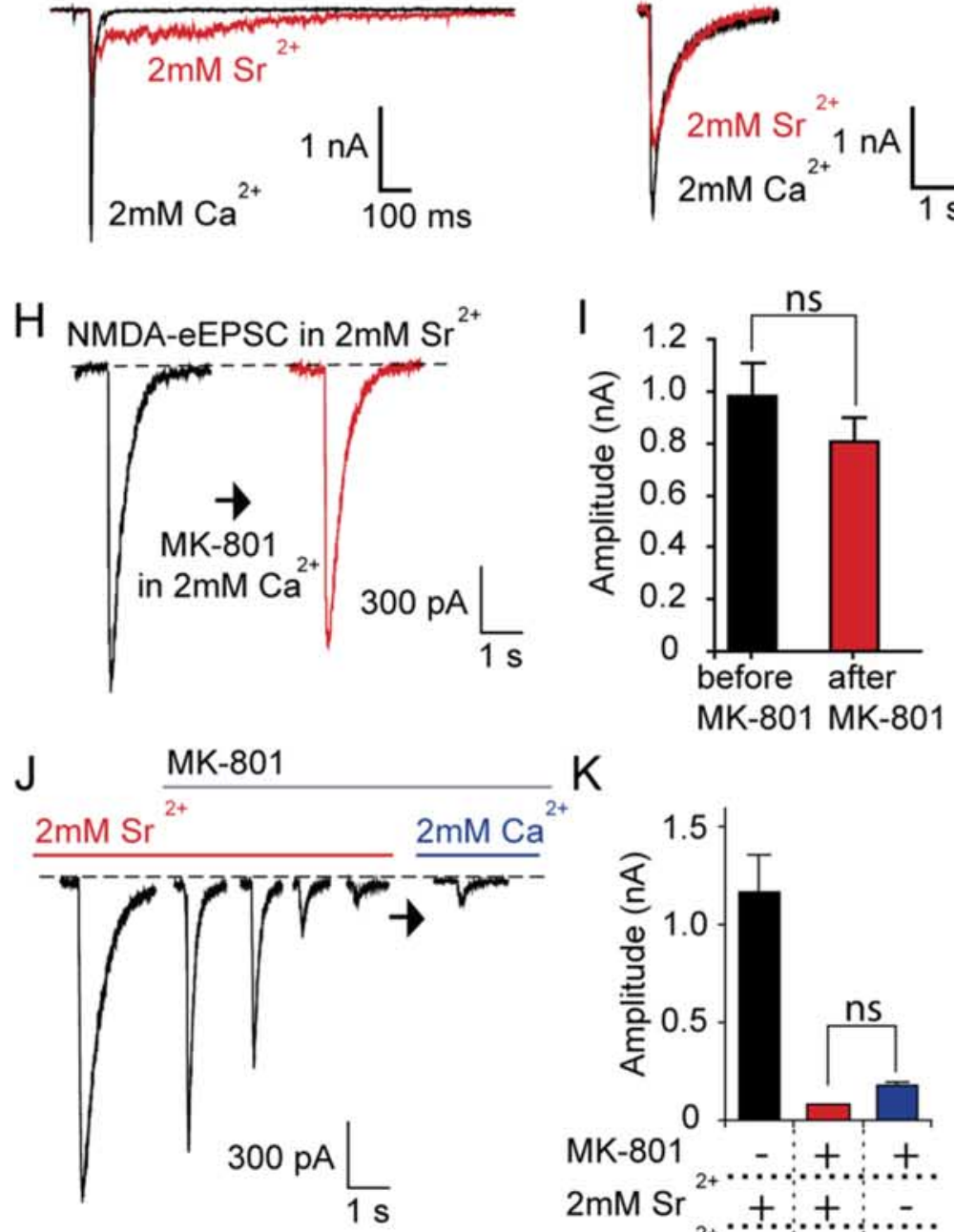

$\mathrm{K}$

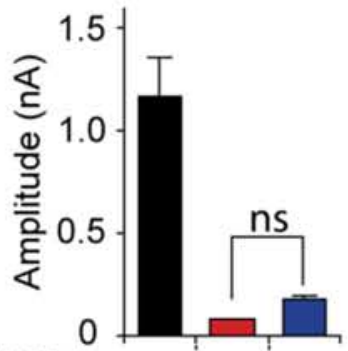

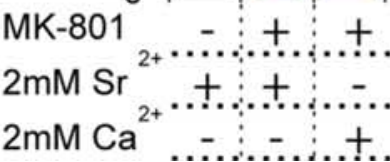

NMDA receptors as spontaneous release. To address this question, we used hippocampal cultures from mice deficient in the synaptic vesicle protein syt-1. Syt-1-deficient synapses release vesicles asynchronously, because syt- 1 is the major sensor for the $\mathrm{Ca}^{2+}$ dependent synchronous release (Geppert et al., 1994; Fernández-Chacón et al., 2001). In syt- $1^{-1-}$ cultures, evoked NMDA-eEPSCs were significantly delayed in their activation and decay, consistent with earlier observations from evoked AMPA-eEPSCs in these cultures (Geppert et al., 1994) (Fig. 9A,B). Interestingly, in parallel with our observations in wild-type rat and mouse cultures, asynchronous NMDA-eEPSCs in $s y t-1^{-1-}$ cultures were unaffected by the $10 \mathrm{~min}$ MK801 treatment at rest (in the presence of TTX) (Fig. 9A,C). In contrast, in syt $-1^{-1-}$ cultures, the frequency of spontaneous NMDA-mEPSCs was twofold higher than

Figure 9. Asynchronous release detected in the absence of synaptotagmin 1 or in strontium is resistant to MK-801 application at rest. $\boldsymbol{A}$, Hippocampal cultures from synaptotagmin1-deficient mice and wild-type littermates are stimulated to elicit NMDA-eEPSCs as in Figure 2. Between the two stimulations, a solution containing TTX plus MK-801 was perfused for $10 \mathrm{~min}$, and NMDA-mEPSCs were recorded (shown in $\boldsymbol{D}$ ). At the end of the 10 min period, MK-801 and TTX were rapidly washed out, and cells were stimulated to evoke an NMDAeEPSC again. $\boldsymbol{B}$, Comparison of rise and decay times of evoked NMDA-eEPSC in wild-type and synaptotagmin-1-deficient cultures [rise times: wild type (wt), $35.6 \pm 2.9 \mathrm{~ms}(n=5)$; syt- $1^{-/-}, 421.8 \pm 35.1 \mathrm{~ms}(n=5) ; p<0.001$; decay times: wt, $255.6 \pm 27 \mathrm{~ms}(n=5) ;$ syt- $^{-1-}, 649.5 \pm 34.7 \mathrm{~ms}(n=$ 5); $p<0.05]$. C, NMDA-eEPSC amplitudes did not show a significant change in wild-type and synaptotagmin-1-deficient cultures [before MK-801: wt, $1795 \pm 261 \mathrm{pA}(n=5)$; syt- $^{-/-}, 700 \pm 84 \mathrm{pA}(n=5) ;$ after MK-801: wt, $1656 \pm$ $420 \mathrm{pA} ;$ syt-1 ${ }^{-/-}, 583 \pm 15 \mathrm{pA}\left(\mathrm{wt}, p>0.7 ;\right.$ syt $-1^{-/-}, p>$ $0.2)]$. D, NMDA-mEPSC traces from the same experiments as shown in $\boldsymbol{A}$. $\boldsymbol{E}$, The time constant of MK-801 block is significantly faster in synaptotagmin-1-deficient cultures ( $p<$ 0.02 ). For wt, $\tau=19 \mathrm{~s}$, and for syt- $1^{-/-}, \tau=8 \mathrm{~s}$. Inset, Baseline charge transfer per $10 \mathrm{~s}$; wt, $10.4 \pm 4 \mathrm{pC} / 10 \mathrm{~s}$; syt-1 ${ }^{-1-}, 30.2 \pm 18 \mathrm{pC} / 10 \mathrm{~s} ;{ }^{*} p<0.05 . \boldsymbol{F}, \mathbf{G}, \mathrm{Sr}^{2+}$ dramatically desynchronizes evoked AMPA-eEPSC but not NMDAeEPSC currents in wild-type cultures. $\boldsymbol{H}, \boldsymbol{I}$, Wild-type hippocampal cultures are stimulated to elicit NMDA-eEPSCs, as in $A$, in the presence of $2 \mathrm{~mm} \mathrm{Sr}^{2+}$ instead of $\mathrm{Ca}^{2+}$. Between the two stimulations, NMDA-mEPSCs were recorded in $\mathrm{Ca}^{2+}$ as in $\boldsymbol{A}$ and $\boldsymbol{B}$. NMDA-eEPSC amplitudes did not show a significant change [before MK-801, $978 \pm 128 \mathrm{pA}(n=5)$; after MK-801, $803 \pm 54 \mathrm{pA} ; p>0.2] . J, K$, Wild-type hippocampal neurons are stimulated to elicit NMDA-eEPSC in the presence of $2 \mathrm{~mm}$ $\mathrm{Sr}^{2+}$ at $0.1 \mathrm{~Hz}$ until a stable baseline is achieved, MK- 801 is then perfused and stimulation is continued for another $3 \mathrm{~min}$ until the responses are blocked. $\mathrm{Sr}^{2+}$ is then washed out, and $\mathrm{Ca}^{2+}$ is washed in, and evoked responses are measured again to check for any recovery. The depressed evoked NMDAeEPSCS did not recover significantly [before MK-801, $1202.5 \pm 199.5 \mathrm{pA}(n=5)$; after 3 min stimulation in MK-801, $78.7 \pm 6 \mathrm{pA}$; after $\mathrm{Sr}^{2+}$ is replaced with $\mathrm{Ca}^{2+}$, $182.5 \pm 15 \mathrm{pA} ; p>0.1]$. 
that of controls (Pang et al., 2006), and accordingly, their rate of MK-801 block was also significantly hastened compared with cultures from littermate controls (Fig. 9D,E) (wild type, $\tau=19 \mathrm{~s}$; syt $-1^{-/-}, \tau=8 \mathrm{~s}$ ). These findings suggest that asynchronous release events detected in the absence of syt-1 activate that same set of NMDA receptors as synchronous events under control conditions. This result agrees with earlier studies showing that synchronous and asynchronous release originate from the same set of vesicles albeit with different release kinetics (Otsu et al., 2004; Sakaba, 2006). Furthermore, the increase in mEPSCs detected in syt-1 $1^{-/}$synapses is independent of the alteration in synchronicity of release seen in the absence of syt-1.

Asynchronous release can also be triggered in wild-type neurons when $\mathrm{Sr}^{2+}$ is used instead of $\mathrm{Ca}^{2+}$ as the charge carrier to elicit neurotransmitter release (Dodge et al., 1969). Therefore, $\mathrm{Sr}^{2+}$ is commonly used in experimental settings where quantal events associated with a specific stimulated input onto a neuron are examined (Oliet et al., 1996). In rat hippocampal cultures, substitution of $\mathrm{Ca}^{2+}$ with $\mathrm{Sr}^{2+}$ resulted in a prominent asynchronous component of the AMPA-eEPSC (Fig. 9F). In contrast, the properties of NMDAeEPSCs were not substantially altered, presumably because of the high affinity of NMDA receptors to glutamate and the resulting slow kinetics of NMDAR-mediated currents (Fig. 9G). Perfusing cultures with MK-801 for $10 \mathrm{~min}$ (in $2 \mathrm{mM} \mathrm{Ca}^{2+}$ ) after eliciting a single $\mathrm{Sr}^{2+}$-mediated NMDA-eEPSC did not significantly alter the magnitude of the subsequent $\mathrm{Sr}^{2+}$-mediated NMDA-eEPSC (Fig. 9H,I). However, continued stimulation in the presence of $\mathrm{Sr}^{2+}$ and MK801 for 3 min resulted in substantial inhibition of NMDA-eEPSCs (Fig. 9J), and washing out $\mathrm{Sr}^{2+}$ with $\mathrm{Ca}^{2+}$ did not cause significant recovery (Fig. 9J,K). Together, these findings suggest that, as in the case of syt-1 $1^{-/-}$synapses, $\mathrm{Sr}^{2+}$-triggered asynchronous release does not activate the same NMDA receptors as spontaneous release and NMDA receptors activated by asynchronous and synchronous release overlap.

\section{Discussion}

In this study, we took advantage of MK-801, a high-affinity usedependent blocker of NMDA receptors, and found that there is limited cross talk between the NMDA receptors that are activated in response to spontaneous versus evoked glutamate release. This finding is based on four principle observations: First, electrophysiological experiments in high-density and autaptic hippocampal cultures as well as hippocampal slices showed that use-dependent block of spontaneous NMDA-mEPSCs and evoked NMDA-eEPSCs were mainly independent. In these experiments, MK-801 application caused a rapid block of NMDA-mEPSCs that was not mediated by the block of presynaptic NMDA receptors, because their inhibition had minimal effect on the rate of spontaneous release. Second, once the NMDA receptors that are activated by both evoked and spontaneous release were blocked, NMDA-mEPSCs showed significant recovery at rest without concomitant recovery of NMDA-eEPSCs. Third, MK-801 block induced by brief exogenous NMDA application preferentially affected spontaneous NMDA-mEPSCs and partially spared evoked NMDA-eEPSCs, suggesting that NMDA receptors responding to evoked neurotransmission have a low probability for opening compared with the receptors responding to spontaneous neurotransmission. Finally, modeling glutamate diffusion and NMDA receptor activation showed that synapses with PSDs larger than $0.2 \mu \mathrm{m}^{2}$ could accommodate two populations of NMDA receptors and respond independently to two fusion events.

There are multiple scenarios that may account for these findings. First, spontaneous and evoked fusion events may originate from different synapses, and thus, they may not activate the same set of receptors (Townsend et al., 2003). However, previous studies in hippocampal cultures have documented substantial colocalization of spontaneous and evoked synaptic vesicle recycling in individual synaptic boutons using uptake of fluorescent markers (Murthy and Stevens, 1999; Prange and Murphy, 1999; Murthy et al., 2000; Sara et al., 2005; Groemer and Klingauf, 2007). Furthermore, the same studies have shown that the sizes of the vesicle pools labeled with spontaneous versus evoked uptake of fluorescence probes in a given nerve terminal are strongly correlated (Murthy and Stevens, 1999; Prange and Murphy, 1999; Sara et al., 2005). Therefore, we consider complete segregation of spontaneous and evoked neurotransmitter release into different synapses as unlikely. Accordingly, the optical analysis that we performed in this study showed that at least $79 \%$ of the synapses are capable of both evoked and spontaneous release, although the kinetics of the two forms of release were not correlated in a given synapse. However, a recent study in the retinal bipolar cell presynaptic terminals using total internal reflection fluorescence microscopy showed that spontaneous fusion events were mostly excluded from synaptic ribbons, which comprised the preferential site for evoked fusion (Zenisek, 2008). Therefore, we cannot exclude that some spontaneous and evoked fusion events may occur at different synapses. This possibility is hard to ascertain in our measurements because of two major caveats of our optical analysis. First, selection of fluorescence puncta in dissociated hippocampal cultures typically favors large synapses over small ones. Our current optical imaging results indicate that only a small fraction of synapses ( $\sim 20 \%)$ support spontaneous or evoked transmission at the expense of the other. However, it is likely that this fraction is higher than our estimates because of the inherent bias in fluorescent puncta selection. Accordingly, the model presented in Figure 8 is consistent with the proposal that some small synapses $\left(<0.2 \mu \mathrm{m}^{2}\right)$ may indeed sustain solely evoked or spontaneous release. Second, the selection of fluorescent puncta that correspond to active synapses using $30 \mathrm{~Hz}$ stimulation may bias our results against a population of synapses that may show low levels of spontaneous release without significant evoked release. Nevertheless, our optical analysis is consistent with an earlier study in the frog neuromuscular junction, which found that the level of spontaneous release is relatively uniform across active zones, and the location of spontaneous release corresponded well with the sites of evoked release, although the propensity of evoked release varied widely among active zones (Zefirov et al., 1995). Together with these earlier findings in hippocampal synapses and the frog neuromuscular junction, our results support the premise that spontaneous and evoked release have substantial overlap in their sites of origin, but they do not possess significant correlation with respect to their kinetics. Therefore, if most spontaneous and evoked release events originate from the same synapse, then it can still be meaningful to record frequency of mEPSCs or mIPSCs to determine whether there is a loss or an increase in the number of synapses. However, it may be difficult to correlate this parameter with evoked release probability.

Our findings may also be accounted for by potential differences between fusion pore kinetics or glutamate release profile of spontaneous and evoked fusion events. For instance, in a given synapse, evoked fusion events may reach a higher percentage of receptors, whereas spontaneous fusion events may activate only a small number of receptors (Cull-Candy and Leszkiewicz, 2004), although the two receptor populations overlap. This possibility contradicts several earlier observations. Both forms of fusion have been shown to equally stimulate AMPA receptors (Sun et al., 2002), which have $\sim 100$-fold less affinity for glutamate than NMDA receptors, suggesting that they both can activate a number of receptors albeit below saturating levels (Mainen et al., 1999). In addition, this scenario is hard to reconcile with the mirror experiments presented in Figures 
2-5 of this study; namely, block of evoked or spontaneous fusion events leads to only limited occlusion of each other, regardless of the order at which they were blocked by MK-801.

A third proposal suggests that spontaneous fusion events may occur ectopically (Matsui and Jahr, 2003; Coggan et al., 2005), outside the active zones, as proposed by some earlier work (Colméus et al., 1982). Our findings may partly support this possibility as long as this "ectopic release" occurs at discrete spots and activates a clustered set of adjacent receptors. The fact that the kinetics of spontaneous and evoked quantal events match under most circumstances (Diamond and Jahr, 1995; Isaacson and Walmsley, 1995; Van der Kloot, 1996; Wall and Usowicz, 1998; Sun et al., 2002) makes a diffuse form of ectopic release an unlikely option to account for our observations. Furthermore, the rapidity of MK-801 block of NMDA-mEPSCs is consistent with the premise that spontaneous fusion events occur in discrete sites, thus repetitively activating a cluster of receptors rather than fusing at sites diffusely distributed along an axon.

The last possibility is that evoked and spontaneous fusion sites are compartmentalized within a single synapse, presumably in the vicinity of a given active zone, thus activating receptors in different subdomains of the PSD. We think this last model brings together the "different synapses" and ectopic release models in one scheme that could account for our data as well as earlier observations (Townsend et al., 2003). This proposal is also supported by the quantitative model we presented in Figure 8, which suggest that medium to large $\left(>0.2 \mu \mathrm{m}^{2}\right.$ area) synapses can easily accommodate independent signaling via spontaneous and evoked release with some geometric constraints. However, our data does not exclude the possibility that small synapses $\left(<0.2 \mu \mathrm{m}^{2}\right.$ area $)$, which may be below the resolution of our optical experiments, can support spontaneous or evoked release exclusively and contribute to our electrophysiological observations. Accordingly, previous work showed that single vesicle fusion events activate only a small number of NMDA receptors (approximately three) that typically comprise $<40 \%$ of the total number of NMDA receptors per postsynaptic site (Nimchinsky et al., 2004). Therefore, we think that there is sufficient latitude for nonoverlapping activation of NMDA receptors within a single synapse by evoked and spontaneous release events.

\section{Probing the properties of asynchronous release}

The findings discussed above gave us an opportunity to address a key question on the role of synaptotagmin 1 in controlling neurotransmitter release. Taking advantage of the differential activation of NMDA receptors by spontaneous and evoked release events, we could show that asynchronous release events still maintained the properties of synchronous evoked transmission by activating a set of NMDA receptors distinct from spontaneous events. In the absence of synaptotagmin 1 , spontaneous release rate was significantly increased. Thus, the increase in spontaneous release and loss of release synchrony seen in syt-1-deficient synapses are separable phenotypes suggesting a dual role for synaptotagmin 1 in regulation of fusion. Furthermore, the asynchronous release elicited in the presence of $\mathrm{Sr}^{2+}$ was also selective in its ability to activate a set of NMDA receptors distinct from spontaneous events and shared with $\mathrm{Ca}^{2+}$-evoked release. Therefore, our results support the premise that asynchronous unitary events detected in $\mathrm{Sr}^{2+}$ provide a more accurate picture for the quantal properties of evoked release (Oliet et al., 1996).

\section{Implications for neuronal signaling}

In addition to their implications for the analysis of unitary neurotransmission, the findings we present here suggest a potential divergence in signaling triggered-by-evoked versus spontaneous activa- tion of postsynaptic neurotransmitter receptors. Although, here, we did not detect a significant difference between the compositions of NMDA receptors activated by the two forms of release, this observation does not exclude differences in the downstream events triggered by the two sets of NMDA receptors. In future experiments, it will be important to test whether other postsynaptic receptor types that respond to different neurotransmitters follow the same premise. In addition, it will be critical to examine the structural determinants of this putative functional compartmentalization within synapses and also investigate whether differential activation of receptors with spontaneous and evoked forms of fusion leads to activation of distinct signaling cascades in target neurons (Sutton et al., 2007).

\section{References}

Chadderton P, Margrie TW, Häusser M (2004) Integration of quanta in cerebellar granule cells during sensory processing. Nature 428:856-860.

Coggan JS, Bartol TM, Esquenazi E, Stiles JR, Lamont S, Martone ME, Berg DK, Ellisman MH, Sejnowski TJ (2005) Evidence for ectopic neurotransmission at a neuronal synapse. Science 309:446-451.

Colméus C, Gomez S, Molgó J, Thesleff S (1982) Discrepancies between spontaneous and evoked synaptic potentials at normal, regenerating and botulinum toxin poisoned mammalian neuromuscular junctions. Proc $\mathrm{R}$ Soc Lond B Biol Sci 215:63-74.

Courant R, Friedrichs K, Lewy H (1928) Über die partiellen differenzengleichungen der mathematischen Physik. Mathematische Annalen 100:32-74.

Cull-Candy SG, Leszkiewicz DN (2004) Role of distinct NMDA receptor subtypes at central synapses. Sci STKE 2004:re16.

Dalby NO, Mody I (2003) Activation of NMDA receptors in rat dentate gyrus granule cells by spontaneous and evoked transmitter release. J Neurophysiol 90:786-797.

Del Castillo J, Katz B (1954) Quantal components of the end-plate potential. J Physiol 124:560-573.

Diamond JS, Jahr CE (1995) Asynchronous release of synaptic vesicles determines the time course of the AMPA receptor-mediated EPSC. Neuron 15:1097-1107.

Dodge FA Jr, Miledi R, Rahamimoff R (1969) Strontium and quantal release of transmitter at the neuromuscular junction. J Physiol 200:267-283.

Dröse S, Altendorf K (1997) Bafilomycins and concanamycins as inhibitors of V-ATPases and P-ATPases. J Exp Biol 200:1-8.

Fatt P, Katz B (1952) Spontaneous subthreshold activity at motor nerve endings. J Physiol 117:109-128.

Fellin T, Pascual O, Gobbo S, Pozzan T, Haydon PG, Carmignoto G (2004) Neuronal synchrony mediated by astrocytic glutamate through activation of extrasynaptic NMDA receptors. Neuron 43:729-743.

Fernández-Chacón R, Königstorfer A, Gerber SH, García J, Matos MF, Stevens CF, Brose N, Rizo J, Rosenmund C, Südhof TC (2001) Synaptotagmin I functions as a calcium regulator of release probability. Nature 410:41-49.

Geppert M, Goda Y, Hammer RE, Li C, Rosahl TW, Stevens CF, Südhof TC (1994) Synaptotagmin I: a major Ca2 + sensor for transmitter release at a central synapse. Cell 79:717-727.

Granseth B, Odermatt B, Royle SJ, Lagnado L (2006) Clathrin-mediated endocytosis is the dominant mechanism of vesicle retrieval at hippocampal synapses. Neuron 51:773-786.

Groc L, Gustafsson B, Hanse E (2002) Spontaneous unitary synaptic activity in CA1 pyramidal neurons during early postnatal development: constant contribution of AMPA and NMDA receptors. J Neurosci 22:5552-5562.

Groemer TW, Klingauf J (2007) Synaptic vesicles recycling spontaneously and during activity belong to the same vesicle pool. Nat Neurosci 10:145-147.

Harris KM, Sultan P (1995) Variation in the number, location and size of synaptic vesicles provides an anatomical basis for the nonuniform probability of release at hippocampal CA1 synapses. Neuropharmacology 34:1387-1395.

Hessler NA, Shirke AM, Malinow R (1993) The probability of transmitter release at a mammalian central synapse. Nature 366:569-572.

Huettner JE, Bean BP (1988) Block of N-methyl-D-aspartate-activated current by the anticonvulsant $\mathrm{MK}-801$ : selective binding to open channels Proc Natl Acad Sci U S A 85:1307-1311.

Isaacson JS, Walmsley B (1995) Counting quanta: direct measurements of transmitter release at a central synapse. Neuron 15:875-884.

Jahr CE (1992) High probability opening of NMDA receptor channels by L-glutamate. Science 255:470-472. 
Jahr CE, Stevens CF (1990) A quantitative description of NMDA receptorchannel kinetic behavior. J Neurosci 10:1830-1837.

Kavalali ET, Klingauf J, Tsien RW (1999) Activity-dependent regulation of synaptic clustering in a hippocampal culture system. Proc Natl Acad Sci U S A 96:12893-12900.

Kimura F, Otsu Y, Tsumoto T (1997) Presynaptically silent synapses: spontaneously active terminals without stimulus-evoked release demonstrated in cortical autapses. J Neurophysiol 77:2805-2815.

Lester RA, Clements JD, Westbrook GL, Jahr CE (1990) Channel kinetics determine the time course of NMDA-receptor-mediated synaptic currents. Nature 346:565-567.

LeVeque RJ (2007) Finite difference methods for ordinary and partial differential equations: steady-state and time-dependent problem. Philadelphia: SIAM.

Liu G, Choi S, Tsien RW (1999) Variability of neurotransmitter concentration and nonsaturation of postsynaptic AMPA receptors at synapses in hippocampal cultures and slices. Neuron 22:395-409.

Mainen ZF, Malinow R, Svoboda K (1999) Synaptic calcium transients in single spines indicate that NMDA receptors are not saturated. Nature 399:151-155.

Matsui K, Jahr CE (2003) Ectopic release of synaptic vesicles. Neuron 40:1173-1183.

McKinney RA, Capogna M, Dürr R, Gähwiler BH, Thompson SM (1999) Miniature synaptic events maintain dendritic spines via AMPA receptor activation. Nat Neurosci 2:44-49.

Mennerick S, Que J, Benz A, Zorumski CF (1995) Passive and synaptic properties of hippocampal neurons grown in microcultures and in mass cultures. J Neurophysiol 73:320-332.

Miesenböck G, De Angelis DA, Rothman JE (1998) Visualizing secretion and synaptic transmission with $\mathrm{pH}$-sensitive green fluorescent proteins. Nature 394:192-195.

Murphy TH, Baraban JM, Wier WG, Blatter LA (1994) Visualization of quantal synaptic transmission by dendritic calcium imaging. Science 263:529-532.

Murthy VN, Stevens CF (1999) Reversal of synaptic vesicle docking at central synapses. Nat Neurosci 2:503-507.

Murthy VN, Sejnowski TJ, Stevens CF (1997) Heterogeneous release properties of visualized individual hippocampal synapses. Neuron 18:599-612.

Murthy VN, Sejnowski TJ, Stevens CF (2000) Dynamics of dendritic calcium transients evoked by quantal release at excitatory hippocampal synapses. Proc Natl Acad Sci U S A 97:901-906.

Nielsen TA, DiGregorio DA, Silver RA (2004) Modulation of glutamate mobility reveals the mechanism underlying slow-rising AMPAR EPSCs and the diffusion coefficient in the synaptic cleft. Neuron 42:757-771.

Nimchinsky EA, Yasuda R, Oertner TG, Svoboda K (2004) The number of glutamate receptors opened by synaptic stimulation in single hippocampal spines. J Neurosci 24:2054-2064.

Oliet SH, Malenka RC, Nicoll RA (1996) Bidirectional control of quantal size by synaptic activity in the hippocampus. Science 271:1294-1297.

Otsu Y, Murphy TH (2003) Miniature transmitter release: accident of nature or careful design? Sci STKE 2003:pe54.

Otsu Y, Shahrezaei V, Li B, Raymond LA, Delaney KR, Murphy TH (2004) Competition between phasic and asynchronous release for recovered synaptic vesicles at developing hippocampal autaptic synapses. J Neurosci 24:420-433.

Pang ZP, Sun J, Rizo J, Maximov A, Südhof TC (2006) Genetic analysis of synaptotagmin 2 in spontaneous and $\mathrm{Ca} 2+$-triggered neurotransmitter release. EMBO J 25:2039-2050.

Paré D, Lebel E, Lang EJ (1997) Differential impact of miniature synaptic potentials on the soma and dendrites of pyramidal neurons in vivo. J Neurophysiol 78:1735-1739.

Paré D, Shink E, Gaudreau H, Destexhe A, Lang EJ (1998) Impact of spontaneous synaptic activity on the resting properties of cat neocortical pyramidal neurons In vivo. J Neurophysiol 79:1450-1460.

Patneau DK, Mayer ML (1990) Structure-activity relationships for amino acid transmitter candidates acting at $N$-methyl-D-aspartate and quisqualate receptors. J Neurosci 10:2385-2399.

Popescu G, Robert A, Howe JR, Auerbach A (2004) Reaction mechanism determines NMDA receptor response to repetitive stimulation. Nature 430:790-793.
Prange O, Murphy TH (1999) Correlation of miniature synaptic activity and evoked release probability in cultures of cortical neurons. J Neurosci 19:6427-6438.

Priestley T, Laughton P, Myers J, Le Bourdellés B, Kerby J, Whiting PJ (1995) Pharmacological properties of recombinant human N-methyl-Daspartate receptors comprising NR1a/NR2A and NR1a/NR2B subunit assemblies expressed in permanently transfected mouse fibroblast cells. Mol Pharmacol 48:841-848.

Rosenmund C, Clements JD, Westbrook GL (1993) Nonuniform probability of glutamate release at a hippocampal synapse. Science 262:754-757.

Rusakov DA, Kullmann DM (1998) Extrasynaptic glutamate diffusion in the hippocampus: ultrastructural constraints, uptake, and receptor activation. J Neurosci 18:3158-3170.

Ryan TA, Reuter H, Smith SJ (1997) Optical detection of a quantal presynaptic membrane turnover. Nature 388:478-482.

Sakaba T (2006) Roles of the fast-releasing and the slowly releasing vesicles in synaptic transmission at the calyx of held. J Neurosci 26:5863-5871.

Sankaranarayanan S, Ryan TA (2001) Calcium accelerates endocytosis of vSNAREs at hippocampal synapses. Nat Neurosci 4:129-136.

Sara Y, Virmani T, Deák F, Liu X, Kavalali ET (2005) An isolated pool of vesicles recycles at rest and drives spontaneous neurotransmission. Neuron 45:563-573.

Saviane C, Silver RA (2006) Fast vesicle reloading and a large pool sustain high bandwidth transmission at a central synapse. Nature 439:983-987.

Scimemi A, Fine A, Kullmann DM, Rusakov DA (2004) NR2B-containing receptors mediate cross talk among hippocampal synapses. J Neurosci 24:4767-4777.

Sun JY, Wu XS, Wu LG (2002) Single and multiple vesicle fusion induce different rates of endocytosis at a central synapse. Nature 417:555-559.

Sutton MA, Wall NR, Aakalu GN, Schuman EM (2004) Regulation of dendritic protein synthesis by miniature synaptic events. Science 304:1979-1983.

Sutton MA, Ito HT, Cressy P, Kempf C, Woo JC, Schuman EM (2006) Miniature neurotransmission stabilizes synaptic function via tonic suppression of local dendritic protein synthesis. Cell 125:785-799.

Sutton MA, Taylor AM, Ito HT, Pham A, Schuman EM (2007) Postsynaptic decoding of neural activity: eEF2 as a biochemical sensor coupling miniature synaptic transmission to local protein synthesis. Neuron 55:648-661.

Thomas CG, Miller AJ, Westbrook GL (2006) Synaptic and extrasynaptic NMDA receptor NR2 subunits in cultured hippocampal neurons. J Neurophysiol 95:1727-1734.

Tovar KR, Westbrook GL (2002) Mobile NMDA receptors at hippocampal synapses. Neuron 34:255-264.

Townsend M, Yoshii A, Mishina M, Constantine-Paton M (2003) Developmental loss of miniature N-methyl-D-aspartate receptor currents in NR2A knockout mice. Proc Natl Acad Sci U S A 100:1340-1345.

Umemiya M, Senda M, Murphy TH (1999) Behaviour of NMDA and AMPA receptor-mediated miniature EPSCs at rat cortical neuron synapses identified by calcium imaging. J Physiol 521:113-122.

Van der Kloot W (1996) Spontaneous and uniquantal-evoked endplate currents in normal frogs are indistinguishable. J Physiol 492:155-162.

Virmani T, Ertunc M, Sara Y, Mozhayeva M, Kavalali ET (2005) Phorbol esters target the activity-dependent recycling pool and spare spontaneous vesicle recycling. J Neurosci 25:10922-10929.

Wall MJ, Usowicz MM (1998) Development of the quantal properties of evoked and spontaneous synaptic currents at a brain synapse. Nat Neurosci 1:675-682.

Waters J, Smith SJ (2002) Vesicle pool partitioning influences presynaptic diversity and weighting in rat hippocampal synapses. J Physiol 541:811-823.

Xu-Friedman MA, Regehr WG (2003) Ultrastructural contributions to desensitization at cerebellar mossy fiber to granule cell synapses. J Neurosci 23:2182-2192.

Zefirov A, Benish T, Fatkullin N, Cheranov S, Khazipov R (1995) Localization of active zones. Nature 376:393-394.

Zenisek D (2008) Vesicle association and exocytosis at ribbon and extraribbon sites in retinal bipolar cell presynaptic terminals. Proc Natl Acad Sci U S A 105:4922-4927.

Zhu Y, Xu J, Stevens CF (2004) High-resolution detection of vesicle recycling at hippocampal synapses using synaptophysin-pHluorin. Soc Neurosci Abstr 30:968.7.

Zucker RS (2005) Minis: whence and wherefore? Neuron 45:482-484. 